\title{
1 Customization and improvement of the LbCas12a-crRNA system for efficient gene
}

\section{2 targeting in plants}

3 Tien Van $\mathrm{Vu}^{1,2,5,{ }^{*}}$, Duong Thi Hai Doan ${ }^{1,5}$, Mil Thi $\operatorname{Tran}^{1,3,5}$, Yeon Woo Sung ${ }^{1}$, Young Jong Song ${ }^{1}$,

4 Jae-Yean $\mathrm{Kim}^{1,4, *}$

$5 \quad{ }^{1}$ Division of Applied Life Science (BK21 FOUR Program), Plant Molecular Biology and

6 Biotechnology Research Center, Gyeongsang National University, Jinju 660-701, Republic of

7 Korea.

$8{ }^{2}$ National Key Laboratory for Plant Cell Biotechnology, Agricultural Genetics Institute, Km 02,

9 Pham Van Dong Road, Co Nhue 1, Bac Tu Liem, Hanoi 11917, Vietnam.

$10{ }^{3}$ Crop Science and Rural Development Division, College of Agriculture, Bac Lieu University, Bac

11 Lieu-97000, Vietnam.

$12{ }^{4}$ Division of Life Science, Gyeongsang National University, 501 Jinju-daero, Jinju 52828, Republic

13 of Korea.

$14{ }^{5}$ These authors contributed equally as co-first authors: Tien Van Vu, Duong Thi Hai Doan, Mil Thi

15 Tran.

$16{ }^{*}$ Correspondence: Jae-Yean Kim: kimjy@gnu.ac.kr (ORCID ID: 0000-0002-1180-6232). Tien Van

17 Vu: tienvu.agi@gmail.com (ORCID: 0000-0002-6369-7664).

18 Keywords: LbCas12a; Gene targeting; Gene editing; Homology-directed repair; SpCas9;

19 geminiviral replicon.

20 Running title: LbCas12a-based gene targeting in tomato.

21 Word count: 5131 
22 Abstract: $149 / 150$

23 Number of Main Figures: 05

24 Number of Supplementary Figures: 12

25 Number of Supplementary Tables: 08

\section{Abstract}

27 Plant gene targeting (GT) can be utilized to precisely replace up to several kilobases of a plant

28 genome. Recent studies using the powerful clustered regularly interspaced short palindromic

29 repeats (CRISPR) and CRISPR-associated (Cas) nucleases significantly improved plant GT

30 efficiency. However, GT for loci without associated selection markers is still inefficient. We

31 previously utilized Lachnospiraceae bacterium Cas12a (LbCas12a) in combination with a

32 replicon for tomato GT and obtained high GT efficiency with some selection markers. In this

33 study, we customize and advance our GT system by using a temperature-tolerant LbCas12a

34 (ttLbCas12a) in combination with various crRNA forms and chemical treatments to suppress the

35 canonical non-homologous end-joining pathway in tomato. Our work demonstrates the

36 significance of the selection of gene scissors, the appropriate design of LbCas12a gRNAs, the

37 use of chemical treatments, and the establishment of favorable experimental conditions for

38 further enhancement of plant HDR to enable efficient GT in tomato.

\section{Introduction}

40 Plant gene targeting (GT) was first reported in 1988 by Paszkowski and coworkers, although

41 that study only obtained low efficiency of targeting ${ }^{1}$, and GT usually requires at least one

42 associated selection marker for the practical achievement of GT events ${ }^{2,3}$. Without any 
43 targeted DSB and with an antibiotic selection marker, the GT efficiency remained extremely

$44 \quad$ low $^{4}$. An important improvement was made to plant GT by introducing DSBs at the targeted

45 sites using a preinserted I-Scel recognition sequence; this approach ultimately enhanced the GT

46 efficiency up to hundreds of fold ${ }^{5,6}$, but the efficiency was still low (i.e., up to $1.83 \%$ ) and

47 required two selection markers. The subsequent development of the first, second and,

48 especially, third generations of site-directed nucleases (SDNs) has revolutionized precision gene

49 editing technology with the easy customization of targeted DSBs at any site of interest for

50 exchanging donor DNA templates ${ }^{7-9}$.

51 Until recently, SDN-based GT efficiency has been significantly enhanced through combinations

52 of SDN complexes and geminiviral replicons, which are autonomously replicative vectors for

53 supplying high doses of homologous DNA template to DSB repair foci ${ }^{10-13}$. Further improvement

54 of plant GT was also possible with the suppression of $\mathrm{CNHEJ}^{14-17}$ or activation of HDR

55 mechanisms ${ }^{18}$ using biological approaches or chemical treatments. Nevertheless, chemical

56 treatments have not been heavily studied in conjunction with CRISPR/Cas-based GT in plants.

57 Overall, the practical GT efficiency with allele-associated selection markers was approximately

$5810 \%$ in most of the "accessible" plants and considerably lower in difficult systems or those with

59 targeted loci lacking selection markers ${ }^{3}$. Therefore, continuous improvement of plant GT

60 remains necessary, especially for applications in less accessible plants.

61 Our previous work showed the significant improvement of plant GT using geminiviral replicons

62 and LbCas12a, rather than SpCas9 ${ }^{12}$. More importantly, the activity of LbCas12a nucleases was

63 more temperature-dependent than that of SpCas9 ${ }^{12,19}$. Recently, a temperature-tolerant

64 LbCas12a mutant (D156R), known as ttLbCas12a, which exhibited significantly improved 

77 GT in tomato.

\section{Results}

cleavage activity and hence gene targeting efficiency, was reported with conventional approaches in Arabidopsis ${ }^{20,21}$. However, further characterization of ttLbCas12a for somatic cell-based GT systems, especially in combination with DNA replicons, has not been undertaken. We hypothesized that ttLbCas12a could be utilized to further improve our replicon-based plant GT system through appropriate customization of its nuclease-crRNA complexes. Chemical treatment for suppressing cNHEJ or activating HDR pathways was also tested and validated for practical GT applications in tomato. In this report, we describe a further comparison of LbCas12a and SpCas9 in inducing GT at the SIANT1 locus. Subsequently, extensive characterization of ttLbCas12a in comparison with LbCas12a is shown at the SIANT1, SIHKT1;2, and SIEPSPS1 loci with or without allele-associated selection markers. Our work demonstrates the significance of utilizing appropriate design of CRISPR/Cas-crRNA complexes, chemical treatments, and favorable experimental conditions to further enhance plant HDR for efficient

\section{NU7441 treatment enhances LbCas12a-based GT efficiency}

A plant genomic DNA DSB may be repaired by two major competing mechanisms, namely, cNHEJ and HDR. The GT approach is based on the HDR mechanism; therefore, to increase its efficacy, blocking the cNHEJ pathway is a good option ${ }^{3}$. A number of studies have been published regarding the uses of chemical treatments for blocking cNHEJ to enhance GT efficiency in mammals ${ }^{16,17,22,23}$. However, only limited information regarding the applications of the chemicals to plant GT is currently available. Therefore, we selected SCR7 (an inhibitor of DNA ligase IV), NU7441 (a DNA-dependent protein kinase (DNA-PKcs) inhibitor), and 
87 KU0060648 (a dual inhibitor of DNA-PKcs and phosphatidylinositol-3 kinase (PI-3K)) to

determine their effects on plant GT. Although no plant homolog of animal DNA-PKcs has been identified to date, alternative DNA-dependent protein kinases might be involved in DNA DSB repair in plants. Previously, a replicon-based CRISPR/Cas-mediated targeted DNA insertion system was successfully developed with SIANT1 as a visible marker ${ }^{10,12}$. We used SpCas9 (pTC217) and LbCas12a (pHR01 and pMR01) carrying GT vectors to assess the effects of chemical treatments.

During the GT reaction performed using the SpCas9-based pTC217 vector, treatment with the DNA ligase IV inhibitor led to an improvement in the GT efficiency compared to the mock control (Fig. 1a, upper panel). The GT efficiency was increased to $34 \%$ with the treatments of either 10 or $50 \mu \mathrm{M}$ SCR7 compared to the mock $0 \mu \mathrm{M}$ treatment or the $1 \mu \mathrm{M}$ SCR7 treatment. However, Fisher's LSD test for comparison of the GT efficiency between the treated concentrations and the mock control did not return significant $p$-values to determine whether the GT enhancement was strong enough (Fig. 1a, upper panel). The data indicate that $10 \mu \mathrm{M}$ SCR7 was the best concentration for SpCas9-based GT in tomato. By contrast, the LbCas12abased pHR01 construct exhibits a decreasing trend from the mock control to the highest SCR7 concentration. There were mild GT efficiency changes among the mock and 1 or $10 \mu \mathrm{M} \mathrm{SCR7}$ treatments. Nevertheless, the GT efficiency was dramatically reduced to $4.140 \pm 0.66 \%$ at 50 $\mu \mathrm{M}$ SCR7 from $6.95 \pm 0.91 \%$ in the mock treatment ( $68 \%$ reduction) (Fig. 1a, bottom panel).

To test the impacts of NU7441 and KU0060648 on CRISPR/Cas-based GT in tomato, we employed both single and multiple replicon systems to carry the SpCas9 or LbCas12a constructs. In the case of SpCas9, the GT efficiency was slightly increased (Fig. 1b, top panel) 
when $0.2 \mu \mathrm{M}$ KU0060648 or $1 \mu \mathrm{M}$ NU7441, which were within the optimal concentration ranges tested in human cells ${ }^{16}$, was applied to NSEL medium (Supplemental Fig. 1) and

111 incubated from days 3 to 8 post-transformation. However, pairwise comparison resulted in $p$ -

112 values that were not small enough to reject the null hypothesis. A similar situation also

113 occurred for the LbCas12a-based single replicon, although NU7441 treatment led to a smaller

114 p-value. In this case, $1 \mu \mathrm{M}$ NU7441 treatment resulted in GT efficiency at $11.07 \pm 1.50 \%$

115 compared to $7.17 \pm 1.67 \%$ of that of the mock control, which represented a 1.54 -fold

116 enhancement (Fig. 1b, middle panel). The effect is considerably more clear with NU7441

117 treatment using the LbCas12a-based multiple replicon system. Blocking cNHEJ with $1 \mu \mathrm{M}$

118 NU7441 significantly increased the multireplicon-based GT efficiency from $11.01 \pm 0.93 \%$ in the

119 mock control to $15.89 \pm 1.15 \%$, representing an approximately 1.44 -fold change (Fig. $1 \mathrm{~b}$,

120 bottom panel). There was almost no GT efficiency change in the case of KU0060648 treatment

121 with pMR01.

122 Silver nitrate treatment enhances GT efficiency and purple shoot regeneration

123 Recently, polyamines, such as putrescine, spermidine, and spermine, were shown to enhance

124 HDR by facilitating RAD51-mediated homologous strand annealing and synaptic complex

125 formation ${ }^{24}$. We added $1 \mathrm{mM}$ putrescine, spermidine, or spermine to the NSEL medium and

126 incubated the transformed explants for 5 days after cocultivation with agrobacteria carrying the

127 pMR01 plasmid. In contrast to the observations made in animals, no improvement in GT

128 efficiency was achieved by any of the polyamines in the two replicates. In fact, the GT efficiency

129 was reduced with supplementation with longer chain polyamines (i.e., spermidine and

130 spermine) (Supplemental Fig. 2). We also observed a higher proliferation of the purple GT calli 
131 and a delay in shoot formation from the explants treated with spermidine or spermine

132 compared to the mock control. We surmised that direct supplementation with synthetic

133 polyamines, especially spermidine and spermine, at high concentrations might not facilitate

134 organ regeneration.

135 In tissue culture, silver nitrate $\left(\mathrm{AgNO}_{3}\right)$ was used as a regulator of ethylene action ${ }^{25}$ that

136 resulted in the enhancement of somatic embryogenesis ${ }^{26,27}$. Silver nitrate was also shown to

137 stimulate the activity of arginine decarboxylase (ADC; EC 4.1.1.9), one of the key enzymes of

138 putrescine biosynthesis ${ }^{28}$. We hypothesized that adding $\mathrm{AgNO}_{3}$ into the tissue culture media

139 may help enhance polyamine synthesis and somatic embryogenesis. Using the pMR01 vector

140 for this experiment, we treated the transformed explants with $30 \mu \mathrm{M} \mathrm{AgNO}$ for 5 days on NSEL

141 medium. In two replicates, we observed a significant increase in the number of purple GT spots

142 per explant compared to that of the mock control (Supplemental Fig. 3a). More importantly,

143 the addition of $\mathrm{AgNO}_{3}$ stimulated shoot regeneration from purple calli (Supplemental Fig. 3b),

144 although it required more time due to the enhancement of callus proliferation.

145 LbCas12a-based GT is superior to the SpCas9-based GT

146 In our previous report, LbCas12a was shown to mediate GT more effectively than the SpCas9

147 system. The comparisons were conducted at the SIANT1 locus under various experimental

148 conditions ${ }^{12}$. However, the comparison using the replicon-based pTC217 and pHR01 constructs

149 might have flawed parameters, such as slightly different SSN-mediated binding and cutting sites

150 and donors. Furthermore, the promoter and terminator driving the expression of SpCas9 and

151 LbCas12a might also contribute to the differences. To better characterize and compare the GT

152 performance of SpCas9 and LbCas12a, we designed a GT system with SIANT1 cutting sites that 
153 are accessible to both nucleases (Fig. 2a and Supplemental Fig. 4) and the same promoter and 154 terminator to drive the transcription of Cas nucleases. The comparisons were conducted using 155 single gRNA (sgR2 ${ }^{\text {ANT1 }}$ vs. crR1.23 $3^{\text {ANT1 }}$ and crR3.20 ${ }^{\text {ANT1 }}$, sgR3 ${ }^{\text {ANT1 }}$ vs. crR3.23 ${ }^{\text {ANT1 }}$ ) (Fig. $2 b$ and Data $156 \mathrm{~S} 1)$. Each of the GT tools was expressed from both the replicon and T-DNA to compare the GT 157 efficiency of the two delivery methods (Fig. 2b). Our data demonstrate the superiority of the 158 replicon system compared to the T-DNA in plant GT, as the GT efficiencies were enhanced 5-8159 fold with the replicons compared to that of the T-DNA tools (Fig. 2c and Supplemental Fig. 5).

160 Moreover, in keeping with our previous data ${ }^{12}$, all of the LbCas12a-based GT constructs, except 161 the 20-nt gRNA, exhibited significantly higher GT efficiencies than the SpCas9-based GT tools 162 (Fig. 2c, $9.53 \pm 0.53 \%$ of pHRC01 vs. $5.1 \pm 0.34 \%$ of pHRC04, $9.53 \pm 0.53 \%$ of pHRC03 vs. $6.73 \pm$ $1630.52 \%$ of pHRC05). These results also indicate that the 23-nt gRNA LbCas12a mediated GT more 164 effectively than the 20-nt gRNA (pHRCO3 vs. pHRC02). Surprisingly, analysis of the indel 165 mutation efficiency of the guide RNAs at the plant stage demonstrated a reverse correlation 166 between the indel mutation efficiency and the GT efficiency at cutting site 1 and cutting site 2

167 (Fig. 2d, pHRCO1 vs. pHRCO4 and pHRC03 vs. pHRC05, Supplemental Fig. 5). This result indicates 168 that a considerably stronger indel mutation activity may negatively affect the GT reactions. 169 Since cNHEJ-mediated indel mutations are favored throughout the life cycle of the cells and 170 HDR is limited to the S-G2 phases ${ }^{3}$, DNA DSBs that form at cell cycles other than S-G2 may lead 171 to permanent modifications at the cutting sites, resulting in the inhibition of any further 172 cleavage at these sites in the HDR-favorable cell cycles. Our data confirmed the stronger activity 173 of SpCas9 in generating DNA DSBs compared to that of LbCas12a in tomato. The highest indel 174 mutation rate was $49.5 \%$ on average with sgR2 ${ }^{\text {ANT1 }}$ (Supplemental Fig. 5). Most of the mutation 
175 traces appear to be deletions (Supplemental Fig. 6). In the experiment, we obtained GT events

176 with a typical purple phenotype due to the overexpression of SIANT1 and the subsequent

177 accumulation of anthocyanin in the plants (Supplemental Fig. 7)

178 ttLbCas12a-based GT efficiency is higher than that of the wild-type variant in the case of the

179 dual crRNA system.

180 The cleavage activity of Cas nucleases was shown to be temperature-dependent, especially in

181 the case of LbCas12a ${ }^{19}$. Previously, we showed the temperature dependency of CRISPR/Cas-

182 mediated GT in tomato ${ }^{12}$. In that case, LbCas12a exhibited considerably better GT support at

183 temperatures as high as $31^{\circ} \mathrm{C}$ compared to that at $19^{\circ} \mathrm{C}$ or $25^{\circ} \mathrm{C}$. This result partially explained

184 why SpCas9 was superior in indel mutation formation compared to LbCas12a at RT. Recently,

185 Puchta's group reported a temperature-tolerant LbCas12a variant that significantly enhanced

186 indel mutation ${ }^{20}$ and $\mathrm{GT}^{21,29}$ efficacy in plants. It is interesting to characterize and compare the

187 ttLbCas12a nuclease using our replicon system and on somatic cells of tomato. We investigated

188 the impacts of single and dual cleavages using 20- or 23-nt gRNA on the GT performance of

189 both the wild-type and ttLbCas12a variants at the well-characterized SIANT1 locus (Fig. 3a;

190 Supplemental Fig. 4 and Data S1). To contribute to the comparison data, another dual crRNA

191 (crR1-2.23 ${ }^{\text {ANT1 }}$ ) was also tested with LbCas12a_gRNA2 (Supplemental Fig. 4), which was

192 previously used by Vu and coworkers ${ }^{12}$. Our data collected from 6 biological replicates were

193 processed and compared using uncorrected Fisher's LSD test. The statistical analysis

194 demonstrated very mild GT efficiency changes among the GT constructs using single crRNAs

195 with both LbCas12a variants (Fig. 3b; pHRC11, pHRC12 and pHRC13 for LbCas12a; and pHRC17,

$196 \mathrm{pHRC18}$ and pHRC19 for ttLbCas12a). There was no significant GT efficiency difference among 
197 the single crRNAs or dual crRNAs with LbCas12a. However, the ttLbCas12a-based dual crRNA constructs (pHRC20, pHRC11 and pHRC22) showed higher GT efficiencies than LbCas12a

199 (pHRC14, pHRC15 and pHRC16, respectively), although the p-values were close to 0.05 and 200 significant (Fig. 3b). The highest difference in GT efficiency was found between ttLbCas12a201 containing pHRC21 (9.74 $\pm 1.49 \%)$ and LbCas12a-containing pHRC15 (6.44 $\pm 0.75 \%)$ with crR1$2023.20^{\text {ANT1 }}$, exhibiting a 1.51-fold change $(p=0.07)$ (Fig. 3b, $\left.c\right)$.

203 In parallel with these experiment, we utilized targeted deep sequencing to analyze the cleavage 204 activity of the nucleases with each of the gRNAs (Fig. 3c) at $10 \mathrm{dpt}$. Due to the large size $205(\sim 0.3 \times 0.3 \mathrm{~cm})$ of the cotyledon explants used in the previous study, the majority of the cells 206 were not in direct contact with the agrobacteria and thus were not transformed efficiently.

207 Therefore, we reduced the size of the cotyledon explant to $\sim 0.1 \times 0.3 \mathrm{~cm}$ (Supplemental Fig. 8) to 208 assess the editing efficiency by targeted deep sequencing. The data collected from two 209 biological replicates showed strong enhancement of indel mutation efficiencies of a gRNA if it 210 was used in a dual crRNA construct, regardless of the LbCas12a variants. The largest

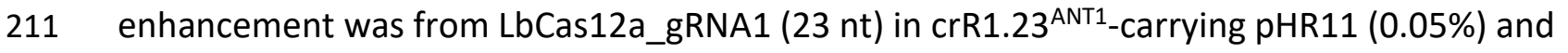
212 crR1-3.23 ${ }^{\text {ANT1 }}$-expressing pHRC16 (1.66\%) in replicate 1, representing a 33.2-fold increase (Fig. 213 3c). The cleavage activity of ttLbCas12a was also higher than that of the WT variant when single

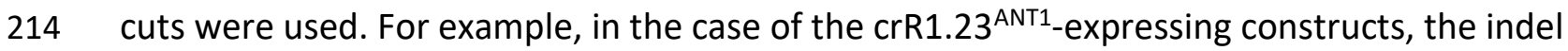
215 mutation efficiency was 5.00- to 7.29-fold increased with ttLbCas12a (pHRC17) compared to 216 LbCas12a (pHRC11) (Fig. 3c). For the cases of the dual crRNAs, ttLbCas12a showed more 217 balanced indel mutation efficiencies between the two gRNAs used in the same construct, 218 especially when dual crR1-3.20 ANT1 with 20-nt gRNAs was examined (Fig. 3c, pHRC21 and 
219 pHRC22 compared to pHRC15 and pHRC16, respectively). This activity might be one of the

220 reasons that better enhancement of GT efficiency was mediated by ttLbCas12a compared to

221 the WT version.

222 ttLbCas12a shows better performance than WT LbCas12a in allele-associated marker-free GT at

223 the SIHKT1;2 and SIEPSPS1 loci

224 To further validate and utilize ttLbCas12a in our research on GT, we compared its performance

225 at the two loci without using any donor-associated selection marker, and no allele-associated

226 selection was employed during the experiments. The salt-tolerant allele SIHKT1;2 (N217D) (Fig.

227 4a) was successfully edited using our LbCas12a-based GT system ${ }^{12}$, although at low efficiency.

228 We introduced two glyphosate-resistant alleles of tomato 5-enolpyruvylshikimate-3-phosphate

229 synthase 1 (SIEPSPS1): (1) T178I and P182S (TIPS allele, corresponding to T103I and P107S in

230 maize, patent no. US6566587B1) ${ }^{30}$ (Fig. 4b) and (2) G177A and A268T (GAAT allele,

231 corresponding to G102A and A193T in maize, patent no. US 6225114 B1) ${ }^{31}$ (Supplemental Fig.

232 9). Extensive comparisons of GT efficiency between LbCas12a and ttLbCas12a were conducted

233 with single crRNAs (crR1.20 ${ }^{\mathrm{HKT} 1 ; 2}$ and $\mathrm{crR} 2.20^{\mathrm{HKT} 1 ; 2}$ ) and dual crRNAs (crR1-2.20 ${ }^{\mathrm{HKT} 1 ; 2}$ and crR1-

$2342.23^{\mathrm{HKT1} ; 2}$ ) (Fig. 4a; Supplemental Fig. 10 and Data S1) at the SIHKT1;2 locus. A T-DNA-based GT

235 construct was also used in parallel with the replicon-based construct and the dual crR1-

$2362.20^{\mathrm{HKT} 1 ; 2}$ construct. Additional comparisons were performed with the dual crRNAs crR1-

$2372.23^{\text {EPSPS1 }}$ and crR1-3.23 ${ }^{\text {EPSPS1 }}$ (Fig. 4b; Supplemental Fig. 10 and Data S1) to replace the TIPS and

238 GAAT alleles, respectively, at the SIEPSPS1 locus.

239 GT efficiency was calculated by GT deep sequencing reads that only showed precise gene

240 editing at both loci in cases of dual crRNAs, although at low efficiencies (Fig. 4c). No GT read 
241 was obtained from the replicon-based single crRNA constructs and the T-DNA-based dual crRNA

242 regardless of the LbCas12a variants. At the SIHKT1;2 locus, the GT efficiency was higher for 243 ttLbCas12a using the dual 20-nt crRNA (crR1-2.20 ${ }^{\mathrm{HKT} 1 ; 2}$ ), but contrasting results were obtained

244 with the longer dual crRNA (crR1-2.23 ${ }^{\mathrm{HKT} 1 ; 2}$ ) (Fig. 4c). However, we obtained higher GT

245 efficiency with the 23-nt dual crRNA (crR1-2.23 ${ }^{\text {EPSPS1}}$ ) for the replacement of the TIPS allele

246 using ttLbCas12a (Fig. 4c). These data indicate that the targeted deep sequencing method may

247 help to identify the GT reads among the constructs, although the read numbers were still too

248 low to be used for statistical comparisons. The indel mutation efficiency obtained from the T-

249 DNA construct was up to 15-fold lower than that of the replicon using the same crR1-2.20 ${ }^{\mathrm{HKT} 1 ; 2}$

250 (Fig. 4c). In this experiment, the indel mutation efficiencies of the single gRNAs were also lower

251 than those of the dual crRNAs. The highest indel mutation activity was obtained with crR1-

$2522.20^{\mathrm{HKT} 1 ; 2}$ and LbCas12a, yielding values of $4.32 \%$ of LbCas12a_gRNA1 and $2.56 \%$ of

253 LbCas12a_gRNA2. The gap between the indel mutation efficiencies of LbCas12a_gRNA1 and

254 LbCas12a_gRNA2 from crR1-2.20 ${ }^{\mathrm{HKT} 1 ; 2}$ was also lower in the case of ttLbCas12a. However,

255 ttLbCas12a did not perform well when it was expressed with the 23-nt dual crRNA (crR1-

$2562.23^{\mathrm{HKT} 1 ; 2}$ ); hence, no GT read was obtained at $10 \mathrm{dpt}$ using the combination (Fig. 4c). In general,

257 between the two replicates, higher indel activity in a construct was correlated with higher GT

258 efficiency.

259 Additional experiments for the exchange of the GAAT allele using the 23-nt dual crRNAs with

260 two (crR1-3.23 $3^{\text {EPSPS1}}$ ) or four cleavage sites (crR1-2.23 $3^{\text {EPSPS1 }}$ and $c r R 2-4.23^{\text {EPSPS1}}$ ) and the

261 LbCas12a variants (Supplemental Figs. 9-10) demonstrated that ttLbCas12a performed better in

262 either case. In triplicate, the highest GT efficiency was obtained with ttLbCas12a-expressing 
constructs (pHRES2.11 compared to pHRES2.9) using the dual gRNA crR1-3.23 $3^{\text {EPSPS1 }}$, up to 0.015

$264 \pm 0.015 \%$ for G177A and $0.018 \pm 0.009 \%$ for A268T (Fig. 5a). Notably, the four gRNA constructs

265 (pHRES2.10 and pHRES2.12) showed a mild reduction in the gRNA1 and gRNA3 indel mutation

266 efficiencies, which were expected to be higher than those of the dual gRNA-expressing

267 constructs due to the synergistic effects of two close cleavage sites (Fig. 5a).

268 To further validate the performance of the LbCas12a variants, we screened and analyzed

269 transformants obtained from the transformation of the LbCas12a- and ttLbCas12a-based

270 constructs for the exchange of the TIPS allele. CAPS screening by Bpil digestion of the PCR

271 products flanking SIEPSPS1-targeted site 1 demonstrated potential TIPS allele-carrying events

272 (Fig. 5b). The Bpil site located 84 bp downstream of the T178 codon was modified during the

273 cloning of the homologous DNA donor through the Golden gate cloning method. Therefore, the

274 undigested bands that appeared on the agarose gel were potentially derived from the TIPS-

275 carrying alleles. Subsequent Sanger sequencing of the purified PCR products from 52

276 transformants per LbCas12a variant and analysis of their.ab1 files by ICE Synthego ${ }^{32}$ identified

277 GT events carrying the TIPS allele (Supplemental Figs. 11-12) at various rates up to 17\% (Fig. 5c,

278 left panel). The average GT efficiency obtained with ttLbCas12a (5.67 $\pm 0.74 \%$ ) was slightly

279 lower than that of the WT version $(4.69 \pm 0.73 \%)$, although the difference was not significant.

280 The difference in plant stage GT efficiency is in contrast with the observation conducted

281 previously by targeted deep sequencing of the $10 \mathrm{dpt}$ cotyledon samples (Fig. 4c). Nevertheless,

282 indel mutation efficiencies at the plant stage correlated with those obtained by targeted deep

283 sequencing (Fig. 5c, right panel). The average indel mutation efficiency was $34.52 \pm 3.30 \%$ with

284 LbCas12a and lower for ttLbCas12a (26.94 $\pm 3.40 \%)(p=0.07$, Student's t-test). Notably, most of 
285 the SIEPSPS1 TIPS allele-carrying GT plants showed no phenotypic changes (Supplemental Fig.

286 12). Several events that contained high indel mutation rates exhibited phenotypic defects

287 compared to WT (Supplemental Fig. 12, pHRES2.7 events \#27 and 28).

288

289

290

291

292

293

294

295

296 of gRNA1 and gRNA2 of the dual crRNA (Fig. 5d).

297

298 The introduction of DNA DSB(s) at the targeted sites was shown to dramatically enhance GT

299 efficiency in plants ${ }^{6}$. DSB repair is dictated by the NHEJ mechanism due to the abundance of

$300 \mathrm{KU70} / 80$ and the other components in the cells ${ }^{3}$. Therefore, the plant HDR efficiency is

301 considerably lower than that of NHEJ. The HDR pathway is more strictly dependent on the cell

302 cycle, and even during favorable S-G2 phases, HDR must also compete with NHEJ to repair DNA

303 DSBs. Previously, accumulated data in animal and plant studies showed the possibility of

304 regulating repair pathway determination or inhibiting cNHEJ components by biochemical or

305 chemical approaches ${ }^{14,16-18,22,23,33,34}$. Among the chemicals that exhibited positive effects on 
HDR or GT in animals, we chose to study several chemicals that inhibit the cNHEJ component(s)

307 to enhance GT efficiency in tomato. One of the alternative strategies for blocking the CNHEJ pathway is to inhibit the DNA-PKcs or

DNA ligase IV was shown to be involved in the last step of cNHEJ-mediated DSB repair to seal the broken ends of DNA DSBs. In a cell-free system, human DNA ligase IV was inhibited by SCR7, a small molecule chemical, by blocking its DNA binding activity ${ }^{34}$. SCR7 at a concentration of 1 $\mu \mathrm{M}$ was shown to enhance CRISPR/Cas9-based GT efficiency up to 5 -fold ${ }^{22}$ or 19 -fold ${ }^{17}$ in mammalian and mouse cells, and $10 \mu \mathrm{M}$ or $60 \mu \mathrm{M}$ SCR7 treatment led to reduced transfection efficiency and cell viability. However, our data demonstrate only a moderate increase in GT efficiency with the SpCas9 construct at toxic levels of SCR7 in animals and no significant enhancement of GT efficiency in the case of LbCas12a (Fig. 1a). Because DNA ligase IV inhibition by SCR7 was irrespective of the DSB configuration ${ }^{34}$, the DNA DSBs generated by either SpCas9 or LbCas12a was not expected to affect the inhibition strength. Since our improved LbCas12abased GT system showed high efficiency under the experimental conditions, the addition of SCR7, which affects the final step of cNHEJ, may not be recognized easily, and the impacts of its toxicity level may be more visible (Fig. 1a). There were limited data regarding the uses of SCR7 for CRISPR/Cas-based GT enhancement in plants; therefore, it is unclear if the SCR7 treatment is species-dependent, since the uptake of SCR7 may be different in plants. The enhancement impacts of SCR7 were also controversial in animals, since studies that used human cell lines or rabbit embryos did not show significant improvement of CRISPR/Cas9- or TALEN-based GT efficiency by SCR7 $7^{18,35,36}$. the other DNA-dependent protein kinases of the PI-3K family by NU7441 ${ }^{37}$ or KU0060648 ${ }^{38}$. 
328 Studies conducted on mammalian cells showed that CRISPR/Cas9-based GT efficiency is

329 enhanced up to twofold by using NU7441 or KU0060648 ${ }^{16}$. However, treatment with $1 \mu \mathrm{M}$

330 NU7441 and 200 nM KU0060648 did not significantly enhance the SpCas9-based GT efficiency

331 under our experimental conditions, although only NU7441 significantly enhanced the LbCas12a-

332 based GT efficiency up to 1.51-fold (Fig. 1b). Since no plant homologs of DNA-PKcs have been

333 identified, these data were surprising but were within our expectations for the existence of

334 other types of plant DNA-dependent protein kinases ${ }^{3}$. More importantly, these data indicate

335 that the inhibitory effects of NU7441 and KU0060648 were selective for the kinase forms and

336 the configurations of DNA DSB ends. Notably, although KU0060648 showed a wider range of

337 inhibition of both DNA-PKcs and PI-3K compared to NU7441, only NU7441 had a positive

338 impact on the LbCas12a GT tools, indicating that the DNA-PKcs targets of KU006648 and

$339 \quad$ NU7441 were distinct.

340 To further improve GT systems, recent studies using polyamines ${ }^{24}$ and silver nitrate, a

341 polyamine biosynthesis regulator, were conducted. In a cell-free assay, polyamines were shown

342 to facilitate RAD51 activities during the formation of synaptic complexes and strand invasion.

343 Depletion of polyamines resulted in the impairment of HDR in mouse hair follicle cells.

344 However, to the best of our knowledge, no direct evidence of the addition of polyamines

345 exerting stimulatory effects on HDR has been reported. Although direct supplementation with

346 polyamines did not affect the CRISPR/Cas-based GT efficiency under our experimental

347 conditions, when we treated the explants with silver nitrate, indirect stimulation of polyamine

348 production using silver nitrate resulted in an increase in GT events, which appeared as purple

349 counting data (Supplemental Fig. 3a). Silver nitrate might also suppress the activities of 
350 ethylene released under the stress induced by agrobacteria and tissue culture processes,

351 leading to the promotion of embryogenic callus proliferation and subsequent somatic

352 embryogenesis (Supplemental Fig. 3b).

353 Previously, we showed that our LbCas12a-based GT system mediated GT more effectively than

354 did SpCas9 complexes ${ }^{12}$. However, direct evidence for the comparison are still required,

355 considering that the accessibility of the cleavage sites and loci by CRISPR/Cas complexes may

356 exhibit different results. Therefore, in this study, we selected the well-characterized SIANT1

357 locus and its two cleavage sites ( 1 and 3 ) to compare the two nucleases using the same or very

358 closed gRNA binding sites (Fig. 2a and Supplemental Fig. 4). The only difference between the

359 SpCas9- and LbCas12a-based vectors was the coding sequence of each of the nucleases, and

360 they were both human-codon optimized (Fig. 2b and Data S1). As expected, the LbCas12-based

361 GT tools outperformed the SpCas9-based replicons at the same cleavage sites (Fig. 2c). Notably,

362 assessment of indel mutation efficiencies of the two systems at the plant stage demonstrated a

363 reverse correlation with the GT efficiencies, as the SpCas9-based indel mutation efficiencies

364 were significantly higher than those of the LbCas12a complexes at the tested cleavage sites (Fig.

$3652 d$ and Supplemental Fig. 5). We reason that the strong cleavage activities of the SpCas9

366 complexes at all the cell cycles may lead to inhibition of the cleavage sites for further recurrent

367 cuts and hence a reduction in the probability of homologous DNA donor-mediated repair by GT

368 in the S-G2 HDR favorable phases. Another important point is that LbCas12a cleaves the

369 targeted sites at a distal side of the TTTV PAM; hence, recurrent cleavages may be possible if

370 the DSB repair of the first cut did not affect the seed sequence located at the proximal side of

371 the $\mathrm{PAM}^{12}$. The view was also supported by a recent study ${ }^{29}$. We cannot exclude the possibility 
372 that the difference in the DNA DSB configurations of SpCas9 (mostly blunt ends) and LbCas12a

373 (cohesive ends) differentially determined repair pathway activation in a spatiotemporal

374 manner, which resulted in the difference in GT efficacy of the two nucleases. In addition, this

375 experiment also indicates that the T-DNA-based gene editing efficiencies were notably low

376 compared to that of the replicon system (Fig. 2c, d) at the same cleavage sites due to its low

377 copy nature.

378 Recently, in attempts to further improve GT efficiency in plants, Puchta's group found that a

379 single mutation (D156R) of LbCas12a significantly improved GT performance compared to WT

380 nuclease 29,39 , especially at the optimal temperature for plant growth. However, a direct

381 comparison of the LbCas12a variants in somatic cell systems has not been reported. Our data

382 not only provide a direct comparison of the two nucleases but also showed their crRNA

383 preference (Fig. 3) in tomato somatic cells for practical applications. There was no significant

384 improvement in GT efficiency using single crRNAs, but ttLbCas12a-base GT might be enhanced

385 with the dual crRNAs, especially with the 20-nt gRNAs at the SIANT1 locus (Fig. 3c, d). The weak

386 improvement in GT efficiency mediated by ttLbCas12a could be explained by the high-

387 temperature experimental conditions applied from day 3 to day 12 throughout our study

388 (Supplemental Fig. 1), which might reduce the low temperature tolerance advantage of

389 ttLbCas12a. Further comparison of the LbCas12a variants at the other two loci, SIHKT1;2 and

390 SIEPSPS1, without using a donor or GT allele-associated selection marker showed better

391 performance of ttLbCas12a with dual 20-nt crRNAs at SIHKT12a (Fig. 4) and dual 23-nt crRNAs

392 at SIEPSPS1 loci (crR1-2.23 $3^{\text {EPSP1 }}$ and crRNA1-3.23 ${ }^{\text {EPSPS1}}$ ) (Figs. 4 and 5a). The data demonstrated

393 the importance of using two neighboring (Fig. 4a, b) or distancing cleavages (Supplemental Fig. 
394 9) for efficient GT, as this approach might offer synergistic effects in the case of neighboring 395 cleavages that lead to considerably higher cutting ${ }^{40}$. In the case of GT alleles that require two 396 distanced sequence modifications, such as the GAAT allele of the SIEPSPS1 loci, two DSBs

397 flanking the targeted sites may ensure simultaneous exchanges of the sequences, since the 398 HDR-based conversion tract generated from each targeted site may not cover the other, due to 399 length limitations ${ }^{29}$.

400 Analysis of the transformants obtained from the GT experiment for exchanging the TIPS allele 401 of SIEPSPS1 revealed high GT efficiency at the plant stage (Fig. 5b, c), although no significant 402 improvement in ttLbCas12a was found at this stage (Fig. 5c). GT events carrying up to $17 \%$ of 403 the GT allele (Fig. 5c) were found with either abnormal or normal phenotypes compared to WT 404 parental plants (Supplemental Fig. 12). The abnormality of the GT events, especially those 405 obtained from the LbCas12a-based construct, was due to the high rates of SIEPSPS1 indel 406 mutation alleles present in the same event. The malfunction of the SIEPSPS1 protein might lead 407 to the inefficiency of aromatic amino acid biosynthesis, which causes phenotypic defects. In this 408 regard, ttLbCas12a, which showed similar GT efficiency but a lower indel mutation rate, may be 409 a better choice for plant GT. Finally, we confirmed the stimulating impacts of $1 \mu \mathrm{M}$ NU7441 on

410 ttLbCas12a-based GT performance, which may enhance the system further and facilitate

411 practical applications for precision crop improvement.

\section{Conclusion}

413 The natural HDR efficiency in plant somatic cells is too low to be utilized for practical 414 applications of GT-mediated plant breeding. Continuous efforts to improve GT performance 
415 have been undertaken for precision crop breeding. In this study, we further improved the

416 LbCas12a-based GT system with the use of chemical treatments ( $1 \mu \mathrm{M}$ of NU7441 and/or

417 AgNO3). The impacts of small molecule chemical treatments on GT have not been well studied

418 in crop plants. Therefore, our data for the assessment of the effects of SCR7, NU7441, and

419 KU0060648 treatments on GT efficiency may help to elucidate their impacts and possible

420 targeted components for HDR pathway regulation in plants.

421 Our data show that LbCas12a outperformed SpCas9 under the same experimental conditions at

422 the SIANT1 loci. Similar effects of the replicon system for GT are also clearly indicated. Despite

423 the milder stimulatory effects on GT performance under our experimental conditions due to

424 the high-temperature protocol, the results of this study indicate that ttLbCas12a might be a

425 good choice for future applications in practical GT in plants. Taken together, the combination of

426 the replicon with ttLbCas12a, double cleavages flanking the modification sequence, and the

427 addition of NU7441 and/or AgNO3 and appropriate temperature conditions are important

428 parameters for the application of GT in future practical applications in precision plant breeding.

429 Methods

430 System design for plant GT in tomato

431 The SIANT1, SIHKT1;2, and SIEPSPS1 loci were used to conduct HDR-based DNA insertion and

432 allele replacement experiments. The chemical treatment experiments used a single replicon

433 (pTC217 and pHR01) and multiple-replicon tool (pMR01) vectors from previous works ${ }^{10,12}$ to

434 target the SIANT1 gene. The SpCas9 containing the pTC217 vector was ordered from Addgene

435 (Plasmid $\# 70018)^{10}$. Further works used the single replicon system as a vector for the delivery of 
436 guide RNA and CRISPR/Cas expression cassettes and GT donor templates in this study (Figure 2a 437 and Data S1).

438 For comparison of the SpCas9 and LbCas12a complexes in GT performance, we designed an 439 HDR-mediated insertion of selection markers at the SIANT1 locus that was well studied in our 440 laboratory ${ }^{12}$. The gRNA binding and cutting sites were selected in a manner that could be used 441 for both SpCas9 (SpCas9_gRNA1 and SpCas9_gRNA2) and LbCas12a (LbCas12a_gRNA1 and 442 LbCas12a_gRNA3) (Figure 2a). We used a single gRNA for each of the GT vectors with either 443 SpCas9 or LbCas12a (Figure 2b). At the cutting site of LbCas12a_gRNA3, two different gRNA 444 lengths (20 and $23 \mathrm{nt}$ ) were tested (Figure $2 \mathrm{~b}$ ). All the editing constructs in the experiment were 445 delivered by Agrobacterium-mediated transformation of tomato cotyledon explants, and two 446 sets of constructs were cloned: one set with the single geminiviral replicon system ${ }^{12}$ for the 447 amplification of homologous DNA donors and the other T-DNA set for comparison.

448 For the assessment and characterization of the activities of ttLbCas12a in GT in comparison 449 with the wild-type version of LbCas12a, similar constructs were designed for targeting the 450 SIANT1 locus with single LbCas12a gRNA expression cassettes. In addition, dual guide RNA 451 expression cassettes combining LbCas12a_gRNA1 (20 and 23 nt) and LbCas12a_gRNA3 (20 and $45223 \mathrm{nt}$ ) were also used for the GT experiments (Figure 3a and Data S1).

453 A similar system for comparison of ttLbCas12a and wtLbCas12a was designed for targeting the 454 SIHKT1;2 and SIEPSPS1 loci (Fig. 4a, b). Single and dual cutting sites were used for SIHKT1;2

455 (Figure 4a, band Data S1). The gRNA lengths (20 and $23 \mathrm{nt}$ ) were also evaluated at the same 
456 locus. For SIEPSPS1, only dual gRNAs $23 \mathrm{nt}$ in length were assessed for GT with the LbCas12a

457 variants (Figure $4 a, b$ and Data S1).

458 In all the GT vectors, the expression of SpCas9 and LbCas12a variants was driven by a long 459 CaMV 35S promoter that contains an intron (Trp1) at the 5'UTR. A copy of AtUBQ10 intron 1

460 was also inserted in the coding sequence of the LbCas12a variants (Data S1), which was tested

461 previously for GT experiments by Vu and coworkers ${ }^{12}$. The crRNA and sgRNA expression

462 cassettes were transcribed with the support of the core sequence of the AtU6 promoter ${ }^{41}$.

463 Agrobacterium-mediated tomato transformation and chemical treatments

464 Agrobacterium-mediated tomato transformation was conducted following our protocol

465 (Supplemental Figure 1) published previously by Vu and coworkers ${ }^{12}$. For the treatment of

466 chemicals, the chemicals at the tested concentrations were added to the NSEL medium. The

467 total treatment time was 5 days (day 3 to day 8 post transformation (dpt)). For targeted deep

468 sequencing, cotyledon samples were collected at $10 \mathrm{dpt}$.

469 Assessment of GT efficiency

470 For assessment of the GT efficiency at SIANT1, purple spot counting was conducted at $21 \mathrm{dpt}$, 471 and purple plants were recorded at the hardening stage. The GT efficiency reflected by the

472 purple spot numbers was calculated with normalization to the SIANT1 overexpression tool that

473 was transformed in parallel with the GT tool. The calculation method was previously explained 474 by Vu and coworkers ${ }^{12}$. 
475 For assessment of the GT efficiency at the SIHKT1;2 and SIEPSPS1 loci, targeted deep

476 sequencing was conducted with thin cotyledon explants collected at $10 \mathrm{dpt}$. Sanger sequencing

477 was performed to screen and validate GT plants.

\section{$478 \quad$ Targeted deep sequencing}

479 Genomic DNAs were isolated from the cotyledon explants using the CTAB method. The MiSeq 480 sequencing service (MiniSeq ${ }^{\mathrm{TM}}$ System, Illumina, USA) was used. MiSeq samples were prepared

481 in three PCRs according to the manufacturer's guidelines with genomic DNAs as templates for

482 the first PCR. The first and second PCRs used primers listed in Supplemental Table 1, whereas

483 the third PCRs were performed with the manufacturer's primers to assign sample IDs. The first

484 PCR primers were designed for binding to the upstream and downstream sequences from the

485 homologous donor sequence junctions to avoid amplifying the donor DNA sequences. The

486 second PCR primers were designed to amplify 150-180-bp flanking the targeted base changes at

487 the targeted sites. High-fidelity DNA Taq polymerase (Phusion, NEB, USA) was used for PCR. The

488 MiSeq raw data FASTQ files were analyzed by the Cas-Analyzer tool ${ }^{42}$. The indel analysis

489 window was set to 5 bases, with a comparison range covering both read ends. The GT efficiency

490 was assessed using the corresponding donor sequence as the input HDR donor sequence.

491 Statistical analysis

492 All comparison experiments were conducted with at least 3 replicates, and data were recorded

493 by purple spot counting, targeted deep sequencing, and plant event screening. Some of the

494 experiments using targeted deep sequencing were conducted in two replicates. The editing

495 data, statistical analysis, and plots were further processed by the MS Excel and GraphPad Prism

496 programs and explained in detail in the legends of figures and/or tables. Pairwise comparison 
497 data were tested with Student's t-test with unequal variance and two-tailed parameters.

498 Similar parameters were applied for multiple comparisons using Fisher's LSD test. A difference

499 was considered to be significant when the statistical tests returned a $p$-value $<0.05$.

500 Acknowledgment

501 We wish to thank Mrs. Ngan Nguyen Thi and Mrs. Jeong Se Jeong for providing valuable 502 technical support to this study. This work was supported by the National Research Foundation

503 of Korea (Grant NRF 2020R1I1A1A01072130, 2020M3A9I4038352, 2020R1A6A1A03044344)

504 and the Program for New Plant Breeding Techniques (NBT, Grant PJ01478401), Rural

505 Development Administration (RDA), Korea.

506 Contribution

507 T.V.V. and J.Y.K. conceived and designed the research. T.V.V., D.T.H.D., M.T.T., Y.W.S., and Y.J.S. 508 conducted experiments. T.V.V., D.T.H.D., M.T.T. and J.Y.K. analyzed data. T.V.V. wrote the 509 manuscript. T.V.V. and J.Y.K. finalized the manuscript. All authors read and approved the 510 manuscript.

\section{Competing interests}

512 The authors declare no competing interests.

\section{References}

514 1. Paszkowski, J., Baur, M., Bogucki, A. \& Potrykus, I. Gene targeting in plants. EMBO J 7, $515 \quad 4021-4026(1988)$.

$5162 . \quad$ Puchta, H. \& Hohn, B. Green light for gene targeting in plants. Proc Natl Acad Sci U S A $517 \quad$ 102, 11961-11962 (2005). 
3. Van Vu, T. et al. Challenges and Perspectives in Homology-Directed Gene Targeting in Monocot Plants. Rice (N Y) 12, 95 (2019).

4. Terada, R., Urawa, H., Inagaki, Y., Tsugane, K. \& lida, S. Efficient gene targeting by homologous recombination in rice. Nat Biotechnol 20, 1030-1034 (2002).

5. Puchta, H., Dujon, B. \& Hohn, B. Homologous recombination in plant cells is enhanced by in vivo induction of double strand breaks into DNA by a site-specific endonuclease. Nucleic Acids Research 21, 5034-5040 (1993).

6. Puchta, H., Dujon, B. \& Hohn, B. Two different but related mechanisms are used in plants for the repair of genomic double-strand breaks by homologous recombination. 93, 5055-5060 (1996).

7. Zhang, Y. et al. Transcription activator-like effector nucleases enable efficient plant genome engineering. Plant Physiol 161, 20-27 (2013).

8. Shan, Q. et al. Targeted genome modification of crop plants using a CRISPR-Cas system. Nat Biotechnol 31, 686-688 (2013).

9. Wright, D.A. et al. High-frequency homologous recombination in plants mediated by zinc-finger nucleases. Plant J 44, 693-705 (2005).

10. Cermak, T., Baltes, N.J., Cegan, R., Zhang, Y. \& Voytas, D.F. High-frequency, precise modification of the tomato genome. Genome Biol 16, 232 (2015).

11. Gil-Humanes, J. et al. High-efficiency gene targeting in hexaploid wheat using DNA replicons and CRISPR/Cas9. Plant J 89, 1251-1262 (2017).

12. Vu, T.V. et al. Highly efficient homology-directed repair using CRISPR/Cpf1-geminiviral replicon in tomato. Plant Biotechnol J (2020).

13. Butler, N.M., Baltes, N.J., Voytas, D.F. \& Douches, D.S. Geminivirus-Mediated Genome Editing in Potato (Solanum tuberosum L.) Using Sequence-Specific Nucleases. Front Plant Sci 7, 1045 (2016).

14. Endo, M., Mikami, M. \& Toki, S. Biallelic Gene Targeting in Rice. Plant Physiol 170, 667677 (2016).

15. Qi, Y. et al. Increasing frequencies of site-specific mutagenesis and gene targeting in Arabidopsis by manipulating DNA repair pathways. Genome Res 23, 547-554 (2013). 
547 16. Robert, F., Barbeau, M., Ethier, S., Dostie, J. \& Pelletier, J. Pharmacological inhibition of DNA-PK stimulates Cas9-mediated genome editing. Genome Med 7, 93 (2015).

17. Maruyama, T. et al. Increasing the efficiency of precise genome editing with CRISPRCas9 by inhibition of nonhomologous end joining. Nat Biotechno/ 33, 538-542 (2015).

18. Song, J. et al. RS-1 enhances CRISPR/Cas9- and TALEN-mediated knock-in efficiency. Nat Commun 7, 10548 (2016).

19. Malzahn, A.A. et al. Application of CRISPR-Cas12a temperature sensitivity for improved genome editing in rice, maize, and Arabidopsis. BMC Biol 17, 9 (2019).

20. Schindele, P. \& Puchta, H. Engineering CRISPR/LbCas12a for highly efficient, temperature-tolerant plant gene editing. Plant Biotechnol J 18, 1118-1120 (2020).

21. Merker, L., Schindele, P., Huang, T.K., Wolter, F. \& Puchta, H. Enhancing in planta gene targeting efficiencies in Arabidopsis using temperature-tolerant CRISPR/LbCas12a. Plant

22. Chu, V.T. et al. Increasing the efficiency of homology-directed repair for CRISPR-Cas9-

23. Zhang, J.P. et al. Efficient precise knockin with a double cut HDR donor after

24. Lee, C.Y. et al. Promotion of homology-directed DNA repair by polyamines. Nat Commun

25. Beyer, E.M. A potent inhibitor of ethylene action in plants. Plant Physiol 58, 268-271 (1976).

568

26. Giridhar, P., Indu, E.P., Vinod, K., Chandrashekar, A. \& Ravishankar, G.A. Direct somatic embryogenesis from Coffea arabica L. and Coffea canephora P ex Fr. under the influence

572 27. Roustan, J.-P., Latche, A. \& Fallot, J. Control of carrot somatic embryogenesis by AgNO3, 573 an inhibitor of ethylene action: Effect on arginine decarboxylase activity. Plant Science 67, 89-95 (1990). 
575 28. Hanfrey, C., Sommer, S., Mayer, M.J., Burtin, D. \& Michael, A.J. Arabidopsis polyamine biosynthesis: absence of ornithine decarboxylase and the mechanism of arginine decarboxylase activity. Plant J 27, 551-560 (2001).

29. Huang, T.-K., Armstrong, B., Schindele, P. \& Puchta, H. Efficient gene targeting in Nicotiana tabacum using CRISPR/SaCas9 and temperature tolerant LbCas12a. Plant Biotechnol J doi: https://doi.org/10.1111/pbi.13546 (2021).

30. Lebrun, M., Sailland, A., Freyssinet, G., DeGryse, E. Mutated 5-enolpyruvylshikimate-3-

31. Eichholtz, D.A., Scott, G.C., Murthy, K.G. Modified gene encoding glyphosate-tolerant 5-

32. Hsiau, T. et al. Inference of CRISPR Edits from Sanger Trace Data. 251082 (2019).

33. Nishizawa-Yokoi, A. et al. A Defect in DNA Ligase4 Enhances the Frequency of TALEN-

34. Srivastava, M. et al. An inhibitor of nonhomologous end-joining abrogates double-strand

35. Gutschner, T., Haemmerle, M., Genovese, G., Draetta, G.F. \& Chin, L. Post-translational

37. Leahy, J.J. et al. Identification of a highly potent and selective DNA-dependent protein

Regulation of Cas9 during G1 Enhances Homology-Directed Repair. Cell Rep 14, 15551566 (2016).

36. Yang, D. et al. Enrichment of G2/M cell cycle phase in human pluripotent stem cells enhances HDR-mediated gene repair with customizable endonucleases. Scientific Reports 6, 21264 (2016). kinase (DNA-PK) inhibitor (NU7441) by screening of chromenone libraries. Bioorg Med Chem Lett 14, 6083-6087 (2004).

38. Munck, J.M. et al. Chemosensitization of cancer cells by KU-0060648, a dual inhibitor of DNA-PK and PI-3K. Mol Cancer Ther 11, 1789-1798 (2012).

39. Merker, L., Schindele, P. \& Puchta, H. Using CRISPR/ttLbCas12a for in planta Gene Targeting in A. thaliana. Curr Protoc Plant Biol 5, e20117 (2020). 
40. Chen, F. et al. Targeted activation of diverse CRISPR-Cas systems for mammalian genome editing via proximal CRISPR targeting. Nat Commun 8, 14958 (2017).

41. Nekrasov, V., Staskawicz, B., Weigel, D., Jones, J.D. \& Kamoun, S. Targeted mutagenesis in the model plant Nicotiana benthamiana using Cas9 RNA-guided endonuclease. Nat Biotechnol 31, 691-693 (2013).

42. Park, J., Lim, K., Kim, J.-S. \& Bae, S. Cas-analyzer: an online tool for assessing genome editing results using NGS data. Bioinformatics 33, 286-288 (2016).

\section{Figure legends}

Fig. 1 Enhancement of GT efficiency by chemical treatments for blocking CNHEJ. a. SpCas9

(pTC217, top panel) and LbCas12a (pHR01, bottom panel)-based GT efficiency obtained from the treatment of different SCR7 concentrations. SCR7 was added to the nonselection medium (NSEL), and the explants were incubated for 5 days before transferring to the selection medium (SEL5). The GT efficiencies were calculated at 21 dpt. b. The impacts of KU0060648 and NU7441 on CRISPR/Cas-based GT. SpCas9-based pTC217 (top panel) and LbCas12a-based pHR01 (middle panel) were cloned with single geminiviral replicons, whereas LbCas12a-based pMR01 (bottom panel) was released from a multireplicon vector. The chemical was added to the nonselection medium (NSEL), and the explants were incubated for 5 days before transferring to the selection medium (SEL5). The GT efficiencies were calculated at $21 \mathrm{dpt}$. Multiple comparisons of the means and plotting were conducted by GraphPad Prism version 9 using one-way ANOVA and Fisher's LSD test. The p-values of each compared mean pair are shown on the top of the bars.

\section{Fig. 2 Performance of the LbCas12a and SpCas9 nucleases in GT-mediated DNA insertion in} tomato. a. Schematic diagram of CRISPR/Cas-based GT processes. The SIANT1 genomic site was cleaved by the CRISPR/Cas complexes at the positions of LbCas12a_gRNA1 and 3 and 
627 SpCas9_gRNA1 and 2, denoted by scissors. Subsequent repairs of the DSBs were conducted

628 with the addition of donor templates that contain upstream homologous arms (corresponding 629 to Donor 3.up and Donor 4.up) and downstream truncated SIANT1 (corresponding to Donors 3

630 and 4.down) of the DSB sites and the inserted sequences containing the kanamycin selection

631 marker (pNOS-NptII-tOCS) followed by a CaMV 35S promoter (35S) for constitutively driving

632 SIANT1 expression. The lengths in bp of the homologous arms are also shown. The distances in

$633 \mathrm{bp}$ among the cleaved sites and the starts and ends of the donor sequences were calculated

634 and illustrated in relation to the ATG start codon of the SIANT1 gene with the A as the +1

635 position. The sequence upstream of the SIANT1 start codon is drawn by the green lines, and

636 purple lines are drawn for the downstream part. The crossing discontinuous lines between the

637 homologous DNA donor and genomic site depict the expected homologous recombination for

638 sequence exchanges. Successful GT would integrate the selection marker and 35S promoter at

639 the DSB sites, thereby supporting event selection and screening by kanamycin antibiotic and

640 purple phenotype. b. Binary vectors used for comparison of SpCas9- and LbCas12a-based GT

641 performance. Each vector contained a homologous donor described in (A) and Data S1, an

642 expression cassette of sgRNA/crRNA and SpCas9 or LbCas12a expression cassette. Two sets of

643 vectors were used: only T-DNA and replicon-based systems for comparison. c. Scatter dot-bar

644 plots showing the GT efficiencies of the tested constructs. $d$, Boxplot showing the indel

645 mutation efficiencies of the GT constructs at the plant stage. The GT efficiencies were

646 calculated at $21 \mathrm{dpt}$. Multiple comparisons of the means and plotting were conducted by

647 GraphPad Prism version 9 using one-way ANOVA and Fisher's LSD test. The p-values of each

648 compared mean pair are shown on the top of the bars. 
Fig. 3 Comparison of GT efficiency between LbCas12a and ttLbCas12a at the SIANT1 locus. a, Binary constructs with the same crRNAs and donors for the assessment of the GT efficiency of LbCas12a (left panel) and ttLbCas12a (right panel). b. Boxplot showing the distributions of GT efficiency among the tools using various crRNAs with LbCas12a and ttLbCas12a. Multiple comparisons of the means of GT efficiency of the constructs using the same sets of crRNAs and donors but with LbCas12a or ttLbCas12a were conducted using Fisher's LSD test, and the pvalues are shown above the compared boxes. c, Indel mutation rates induced by the Cas-crRNA complexes that were assessed at $10 \mathrm{dpt}$ by targeted deep sequencing method. The GT efficiencies are also added for comparison.

\section{Fig. 4 GT performance of the LbCas12a variants at the SIHKT1;2 and SIEPSPS1 loci.}

a-b. Schematic diagrams describing the expected GT processes for exchanging the homologous DNA donor template with the genomic sequence at the SIHKT1;2 (a) and SIEPSPS1 (b) loci. The D217 coding sequence was added during the cloning of the HKTD1 donor for exchange with the N217 sequence of the genomic site. The lengths of homologous arms are shown. Two cutting sites (LbCas12a cutting sites 1 and 2) were planned to support the GT. The reverse and forward primers for amplifying the targeted sites by PCR are shown with black arrows. The I178 and S182 coding sequences were added during the cloning of the EPSPS1D3 donor for exchange with the T178 and P182 sequences of the genomic site. The lengths of homologous arms are shown. Two cutting sites (LbCas12a cutting sites 1 and 2) were used for the GT experiments. The reverse and forward primers for amplifying the targeted sites by PCR are shown with black arrows. In b, LbCas12a cutting site 2 is set as position 1, and the other positions are calculated accordingly. The diagrams were drawn not to their actual scales. c. The GT and indel mutation 
671 efficiencies assessed by targeted deep sequencing. At the SIHKT1;2 locus, four different crRNAs

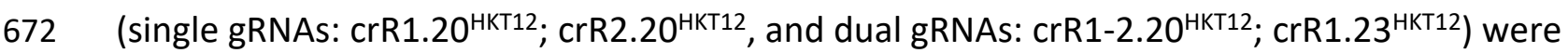

673 used for comparison of the LbCas12a variants in GT performance. A T-DNA vector was also used

674 for comparison with the replicon system. With the SIEPSPS1 gene, only one dual gRNA

675 construct (crR1-2.23 ${ }^{\text {EPSPS1}}$ ) was used with the two LbCas12a variants. Wt: wild-type LbCas12a; tt:

676 ttLbCas12a.

677 Fig. 5 Further assessment of GT performance of the LbCas12a variants at the SIEPSPS1 locus.

678 a. Assessment of GT efficiency by targeted deep sequencing with GT tools using two or four

679 cutting sites with LbCas12a variants at the SIEPSPS1 locus. b-c. Indel mutation and GT

680 efficiencies obtained with the LbCas12a variants at the plant stage. Fifty-two plants of each

681 LbCas12a variant were obtained from the transformation of the GT tool with the crR1-2.23 ${ }^{\text {EPSPS1 }}$

682 expression cassette and used for PCR amplification of the targeted site with the UPEPSPS1-F2

683 and DNEPSPS1-R1 primers. In $\mathbf{b}$, the PCR products were purified and screened for the potential

684 GT allele by Bpil digestion since the Bpil site near the targeted site was modified in the DNA

685 donor sequence. The red arrows indicate potential GT bands. 1-15: Representative

686 transformants obtained from the transformation using the GT construct containing LbCas12a

687 and crR1-3.23 ${ }^{\text {EPSPS1}}$. In c: All the purified PCR products were sequenced by the Sanger method,

688 and the ab1 files were subsequently analyzed by ICE Synthego software to reveal the indel

689 mutation and GT efficiencies. The indel mutation and GT efficiencies of all the samples were

690 statistically analyzed using Student's t-test and plotted by GraphPad Prism version 9. The

691 editing efficiencies (mean \pm SEM) are shown at the bottom of each box. $\mathbf{d}$. Targeted deep

692 sequencing-mediated evaluation of NU7441 impacts on ttLbCas12a-based GT efficiency. 
694 Supplemental Fig. 1 Agrobacterium-mediated transformation protocol used in this work.

695 Supplemental Fig. 2 Effects of polyamine treatment on the GT efficiency.

696 Supplemental Fig. 3 Effects of AgNO3 treatment on purple spot numbers and purple shoot 697 regeneration.

698 Supplemental Fig. 4 Map of SpCas9 and LbCas12a binding sites at the SIANT1 locus.

699 Supplemental Fig. 5 Editing performance of the T-DNA and replicon-based SpCas9 and LbCas12a

700 GT tools at various sites of the SIANT1 locus.

701 Supplemental Fig. 6 Representative indel mutation traces of the GT constructs revealed from

702 ICE Synthego analysis.

703 Supplemental Fig. 7 Representative SIANT1 GT events obtained from the study.

704 Supplemental Fig. 8 Thin slice cotyledon explants for the assessment of editing efficiencies by 705 targeted deep sequencing at $10 \mathrm{dpt}$.

706 Supplemental Fig. 9 Diagram showing the strategy for replacement of two amino acids of the 707 SIEPSPS1 gene.

708 Supplemental Fig. 10 GT constructs for editing the SIHKT1;2 and SIEPSPS1.

709 Supplemental Fig. 11 Representative of the TIPS allele revealed at the plant stage.

710 Supplemental Fig. 12 Representative GT events obtained using the GT tool for TIPS allele 711 replacement. 


\section{Supplemental Tables}

713 Supplemental Table 1. Sequences and primers used in this study.

714 Supplemental Table 2. Impacts of SCR7 on GT efficiency.

715 Supplemental Table 3. GT efficiency obtained from the treatment of RS1 and SCR7.

716 Supplemental Table 4. GT performance revealed from the treatment of KU0060648 and

$717 \quad$ NU7441.

718 Supplemental Table 5. Shoot regeneration after treatment with KU0060648 and NU7441.

719 Supplemental Table 6. Negative impacts of ABA treatment on GT efficiency.

720 Supplemental Table 7. GT efficiency obtained from polyamine treatment.

721 Supplemental Table 8. GT efficiency of the AgNO3 and mock treatments of pMR01. 


\section{Figure 1}

a
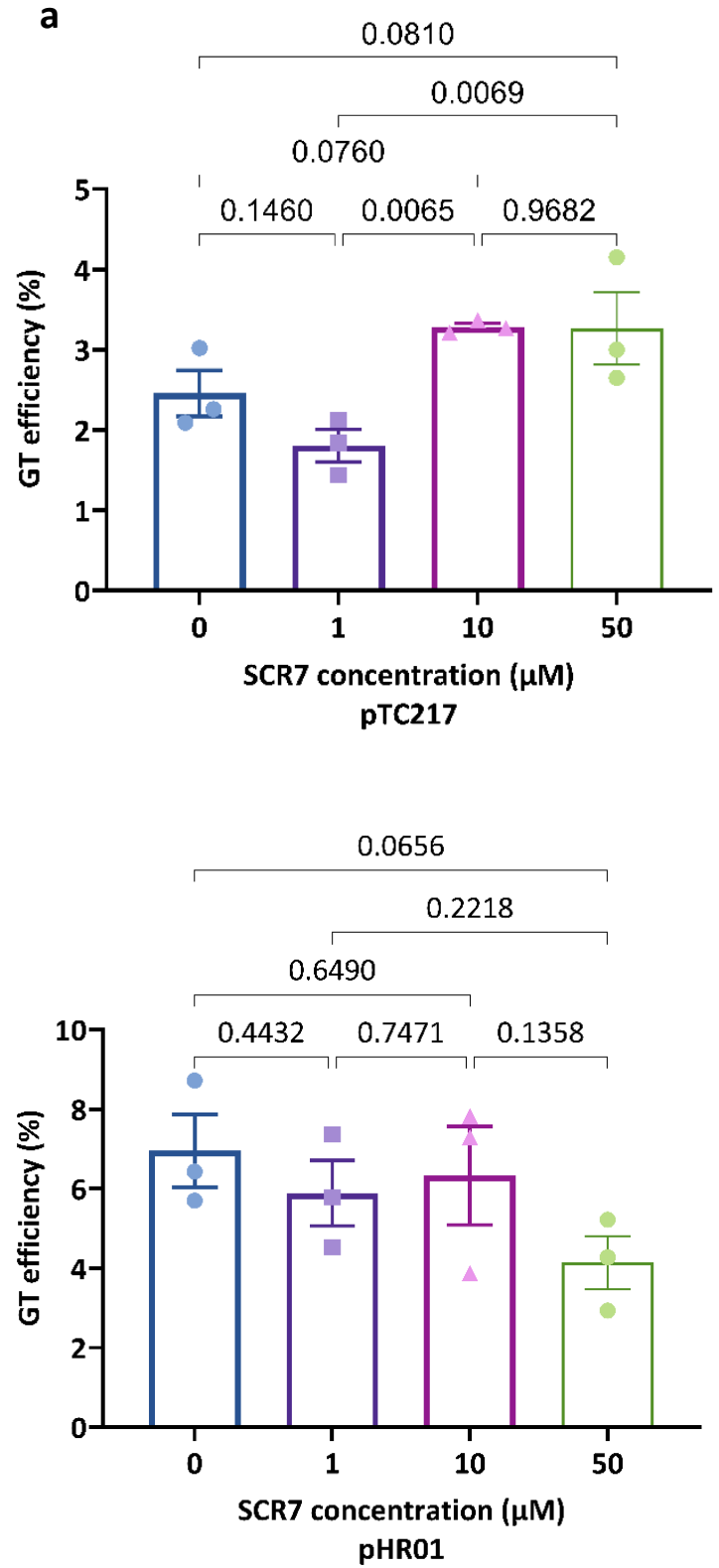

b
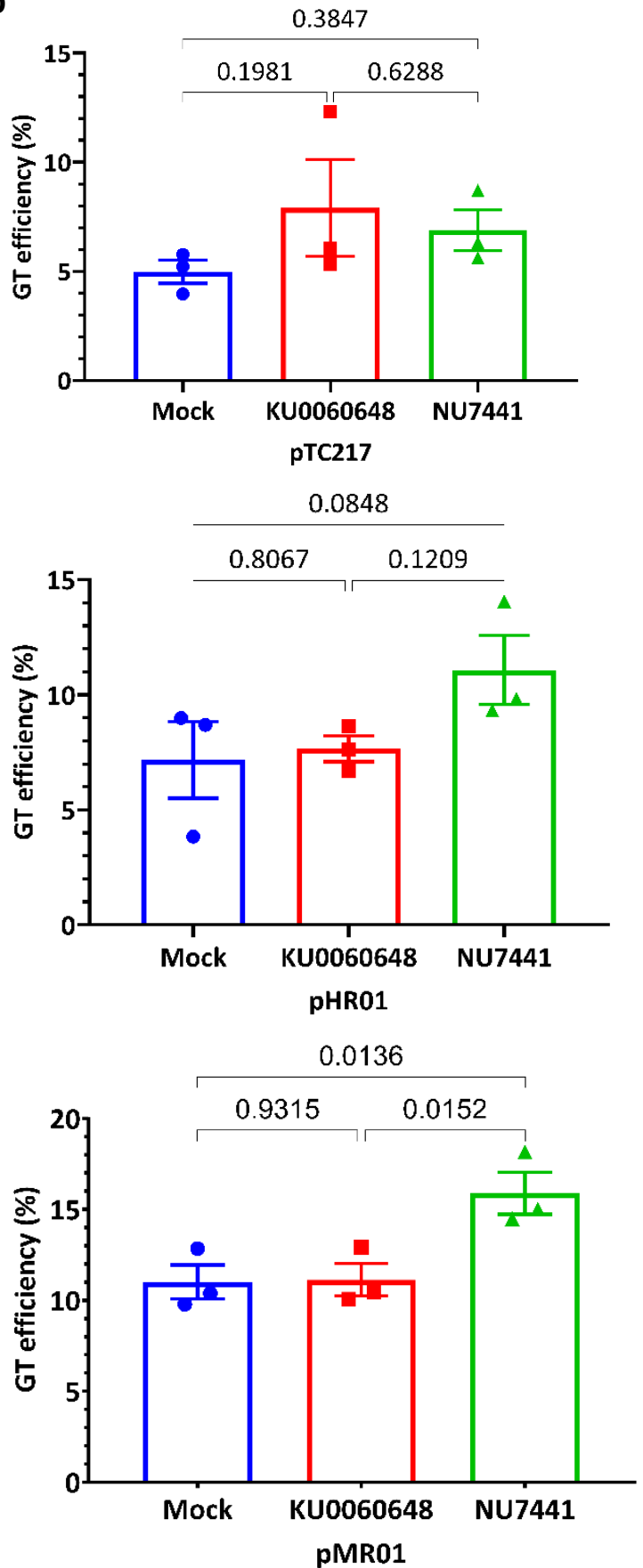
Fig. 1 Enhancement of GT efficiency by chemical treatments for blocking cNHEJ. a. SpCas9 (pTC217, top panel) and LbCas12a (pHR01, bottom panel)-based GT efficiency obtained from the treatment of different SCR7 concentrations. SCR7 was added to the nonselection medium (NSEL), and the explants were incubated for 5 days before transferring to the selection medium (SEL5). The GT efficiencies were calculated at $21 \mathrm{dpt}$. b. The impacts of KU0060648 and NU7441 on CRISPR/Cas-based GT. SpCas9-based pTC217 (top panel) and LbCas12a-based pHR01 (middle panel) were cloned with single geminiviral replicons, whereas LbCas12a-based pMR01 (bottom panel) was released from a multireplicon vector. The chemical was added to the nonselection medium (NSEL), and the explants were incubated for 5 days before transferring to the selection medium (SEL5). The GT efficiencies were calculated at $21 \mathrm{dpt}$. Multiple comparisons of the means and plotting were conducted by GraphPad Prism version 9 using one-way ANOVA and Fisher's LSD test. The $p$-values of each compared mean pair are shown on the top of the bars. 
Figure 2

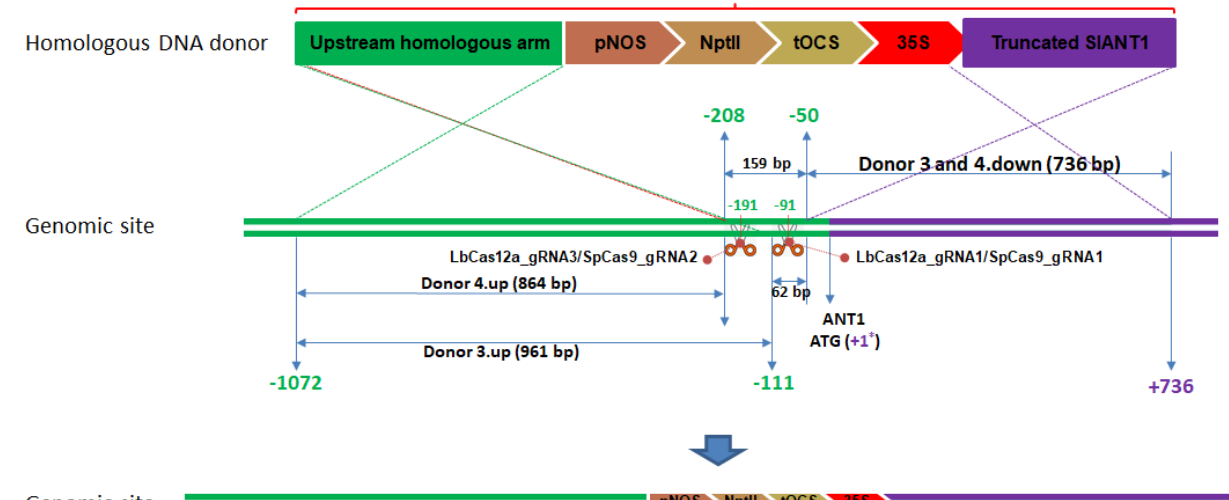

HGT products

ANT1 over expression, purple phenotype

C

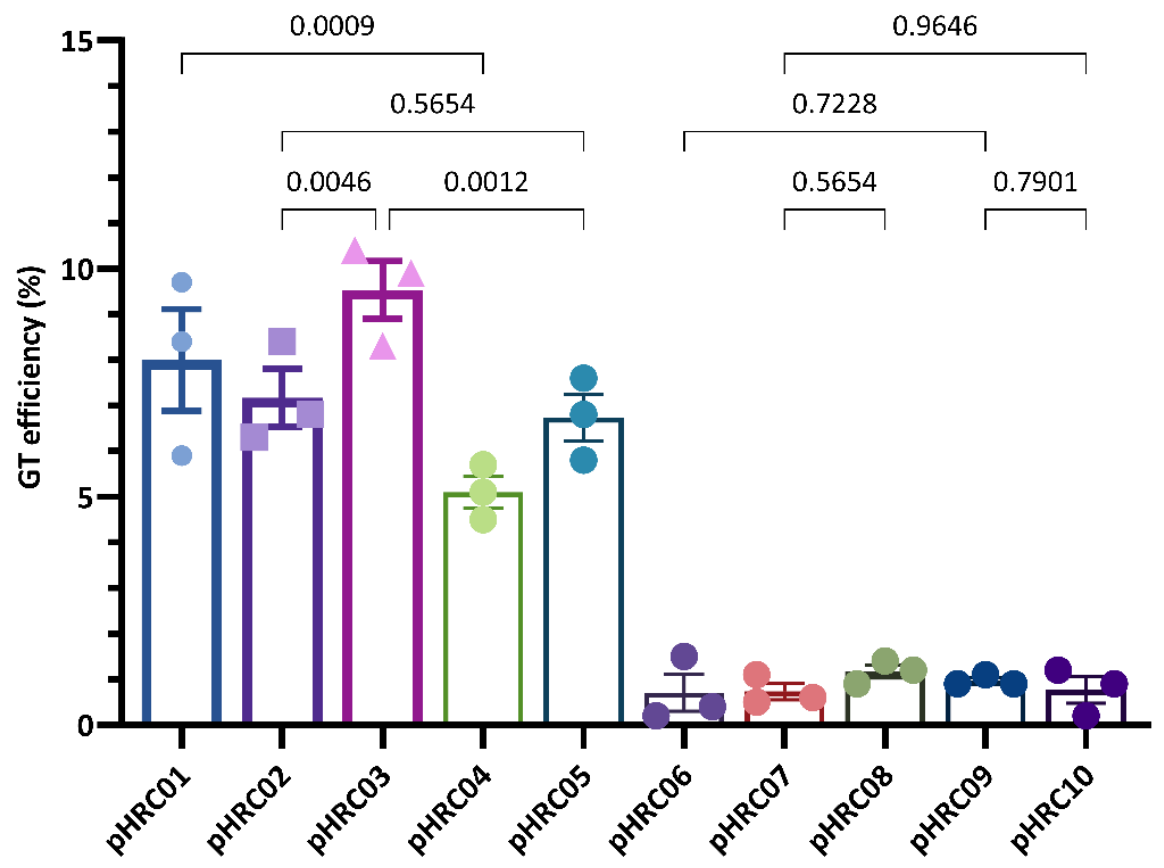

b

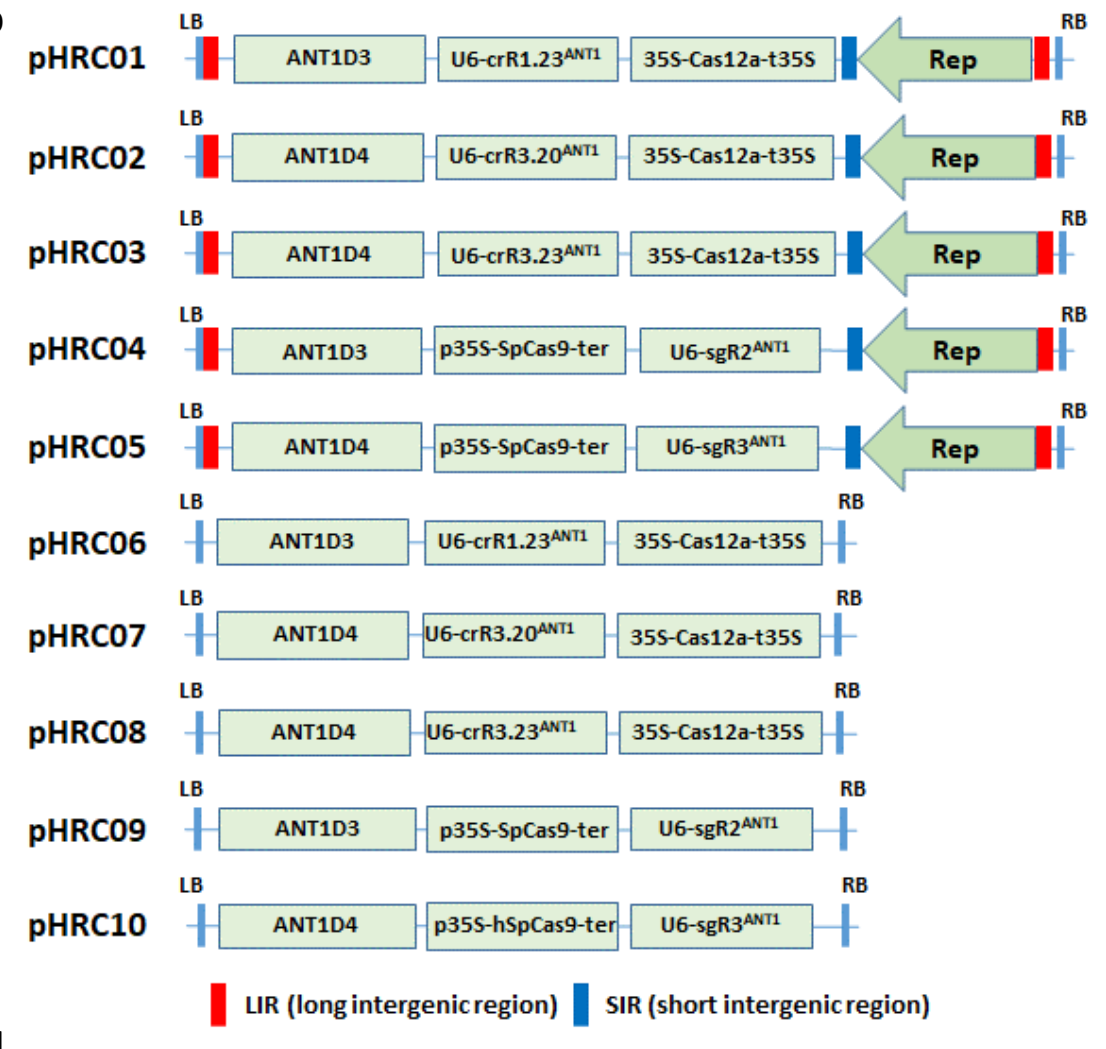

d

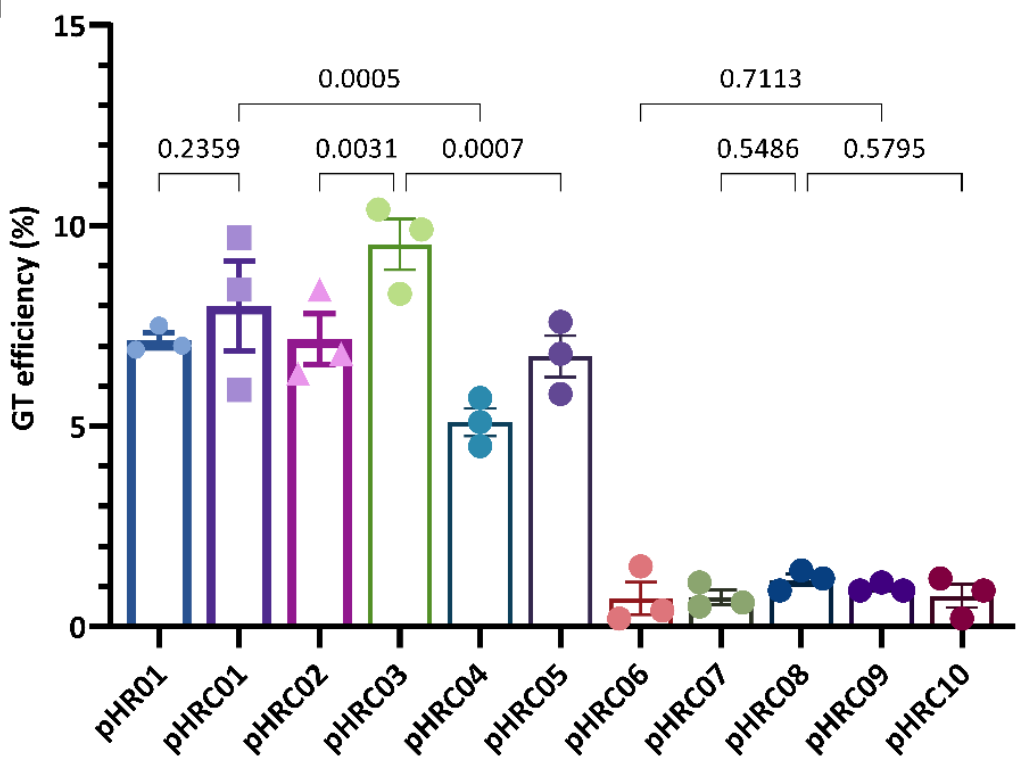


Fig. 2 Performance of the LbCas12a and SpCas9 nucleases in GT-mediated DNA insertion in tomato. a. Schematic diagram of CRISPR/Cas-based GT processes. The SIANT1 genomic site was cleaved by the CRISPR/Cas complexes at the positions of LbCas12a_gRNA1 and 3 and SpCas9_gRNA1 and 2, denoted by scissors. Subsequent repairs of the DSBs were conducted with the addition of donor templates that contain upstream homologous arms (corresponding to Donor 3.up and Donor 4.up) and downstream truncated SIANT1 (corresponding to Donors 3 and 4.down) of the DSB sites and the inserted sequences containing the kanamycin selection marker (pNOS-NptIl-tOCS) followed by a CaMV 35S promoter (35S) for constitutively driving SIANT1 expression. The lengths in bp of the homologous arms are also shown. The distances in bp among the cleaved sites and the starts and ends of the donor sequences were calculated and illustrated in relation to the ATG start codon of the SIANT1 gene with the A as the +1 position. The sequence upstream of the SIANT1 start codon is drawn by the green lines, and purple lines are drawn for the downstream part. The crossing discontinuous lines between the homologous DNA donor and genomic site depict the expected homologous recombination for sequence exchanges. Successful GT would integrate the selection marker and 35S promoter at the DSB sites, thereby supporting event selection and screening by kanamycin antibiotic and purple phenotype. b. Binary vectors used for comparison of SpCas9- and LbCas12a-based GT performance. Each vector contained a homologous donor described in (A) and Data S1, an expression cassette of sgRNA/crRNA and SpCas9 or LbCas12a expression cassette. Two sets of vectors were used: only T-DNA and repliconbased systems for comparison. c. Scatter dot-bar plots showing the GT efficiencies of the tested constructs. d, Boxplot showing the indel mutation efficiencies of the GT constructs at the plant stage. The GT efficiencies were calculated at $21 \mathrm{dpt}$. Multiple comparisons of the means and plotting were conducted by GraphPad Prism version 9 using one-way ANOVA and Fisher's LSD test. The p-values of each compared mean pair are shown on the top of the bars. 


\section{Figure 3}

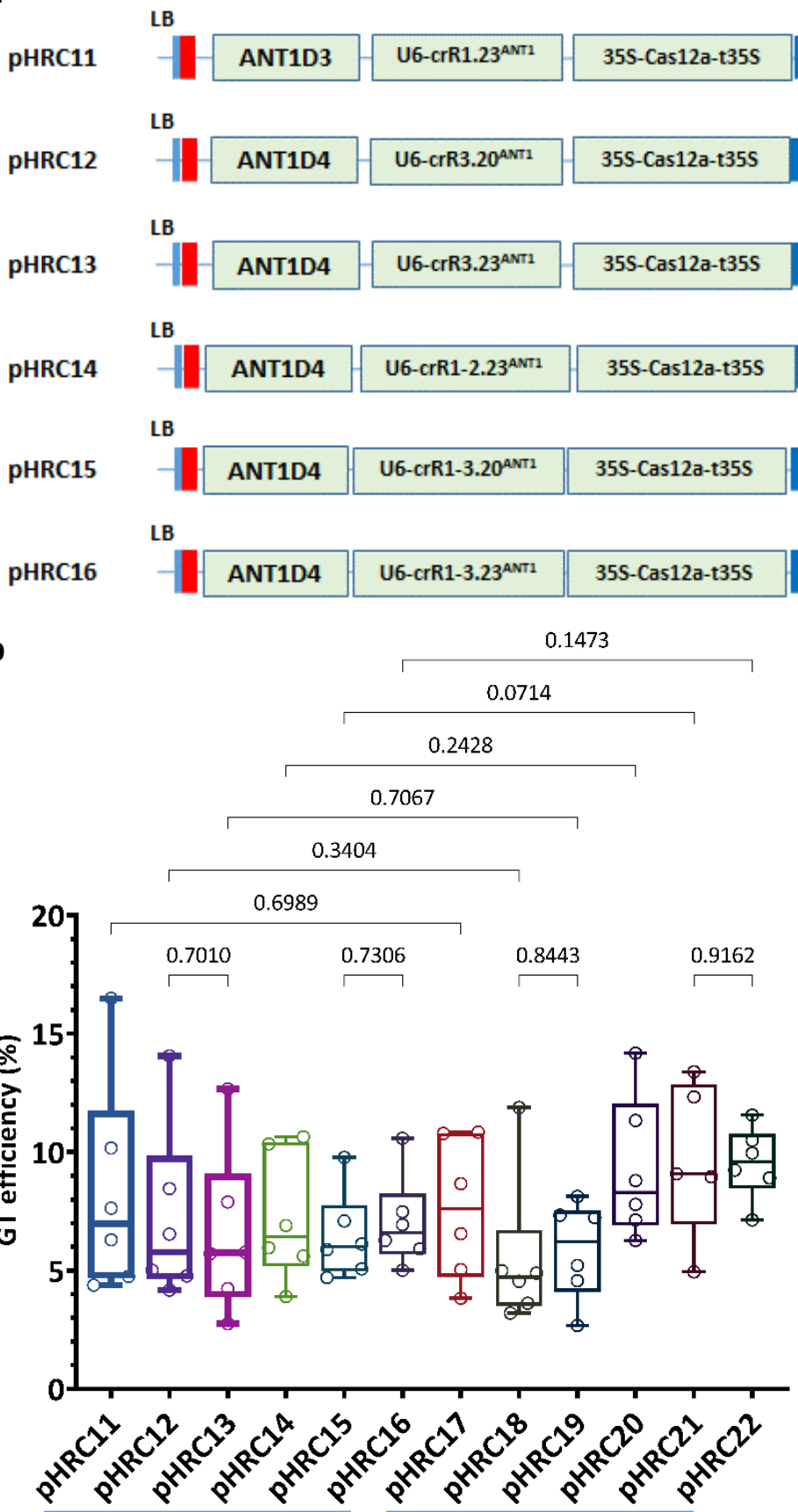

RB
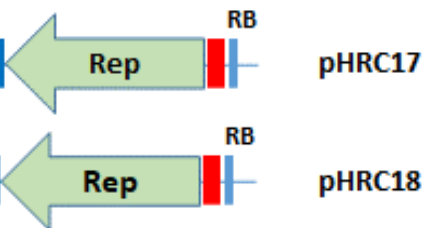

pHRC18

RB

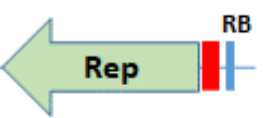

PHRC19

RB

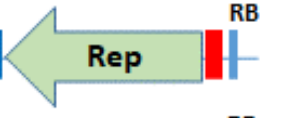

pHRC20

RB

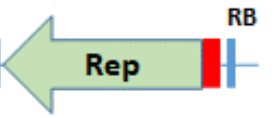

pHRC21

RB

pHRC22
LB

$-$

LB

ANT1D3

U6-crR1.23 $23^{\text {ANT1 }}$

35S-ttCas12a-t35s

$12 \operatorname{Rep}$

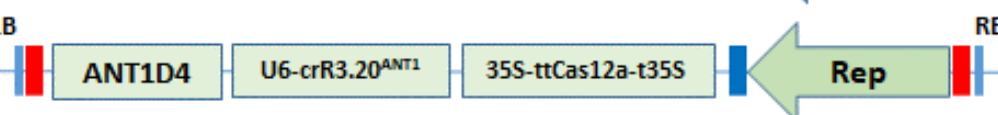

B ANT1D4 U6-crR3.23 ${ }^{\text {ANT1 }}$ 35s-ttCas12a-t35s Rep

RB

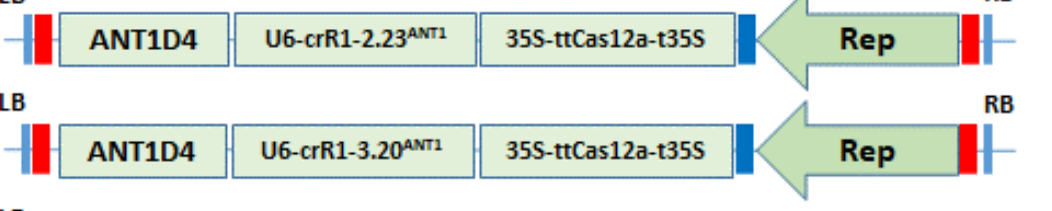

LB ANT1D4 U6-crR1-3.23 ${ }^{\text {ANT1 }}$ 35S-ttCas12a-t35s $\operatorname{Rep}^{\text {RB }}$

LIR (long intergenic region) SIR (short intergenic region)
Construct $\begin{array}{ll}\text { LbCas12 } & \text { ANT1 } \\ \text { a variant } & \text { crRNA }\end{array}$

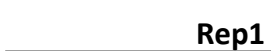

gRNA1

Miniseq Miniseq Miniseq Miniseq Miniseq Miniseq indel rates indel rates indel rates indel rates indel rates indel rates
(\%)

(\%)

(\%)

(\%)

\begin{tabular}{|c|c|c|c|c|c|c|c|c|c|}
\hline pHRC11 & \multirow{6}{*}{ wt } & crR1.23 & 0.05 & - & - & 0.17 & - & - & $8.29 \pm 1.85$ \\
\hline pHRC12 & & crR3.20 & - & - & 0.26 & - & - & 0.74 & $7.17 \pm 1.52$ \\
\hline pHRC13 & & crR3.23 & - & - & 0.05 & - & - & 0.24 & $6.51 \pm 1.42$ \\
\hline pHRC14 & & crR1-2.23 & 0.71 & 0.04 & - & 1.52 & 0.10 & - & $7.23 \pm 1.12$ \\
\hline pHRC15 & & crR1-3.20 & 1.44 & - & 0.84 & 1.91 & - & 1.00 & $6.44 \pm 0.75$ \\
\hline pHRC16 & & crR1-3.23 & 1.66 & - & 0.35 & 2.04 & - & 0.44 & $7.03 \pm 0.79$ \\
\hline pHRC17 & \multirow{6}{*}{$\mathrm{tt}$} & crR1.23 & 0.20 & - & - & 1.24 & - & - & $7.62 \pm 1.21$ \\
\hline pHRC18 & & crR3.20 & - & - & 0.12 & - & - & 1.52 & $5.52 \pm 1.31$ \\
\hline pHRC19 & & crR3.23 & - & - & 0.14 & - & - & 0.85 & $5.86 \pm 0.84$ \\
\hline pHRC2O & & crR1-2.23 & 0.36 & 0.05 & - & 0.83 & 0.12 & - & $9.25 \pm 1.21$ \\
\hline pHRC21 & & crR1-3.20 & 1.34 & - & 1.49 & 1.60 & - & 1.62 & $9.74 \pm 1.49$ \\
\hline pHRC22 & & crR1-3.23 & 1.72 & - & 1.00 & 3.00 & - & 2.31 & $9.55 \pm 0.62$ \\
\hline
\end{tabular}


Fig. 3 Comparison of GT efficiency between LbCas12a and ttLbCas12a at the SIANT1 locus. a, Binary constructs with the same crRNAs and donors for the assessment of the GT efficiency of LbCas12a (left panel) and ttLbCas12a (right panel). b. Boxplot showing the distributions of GT efficiency among the tools using various crRNAs with LbCas12a and ttLbCas12a. Multiple comparisons of the means of GT efficiency of the constructs using the same sets of crRNAs and donors but with LbCas12a or ttLbCas12a were conducted using Fisher's LSD test, and the p-values are shown above the compared boxes. c, Indel mutation rates induced by the Cas-crRNA complexes that were assessed at $10 \mathrm{dpt}$ by targeted deep sequencing method. The GT efficiencies are also added for comparison. 


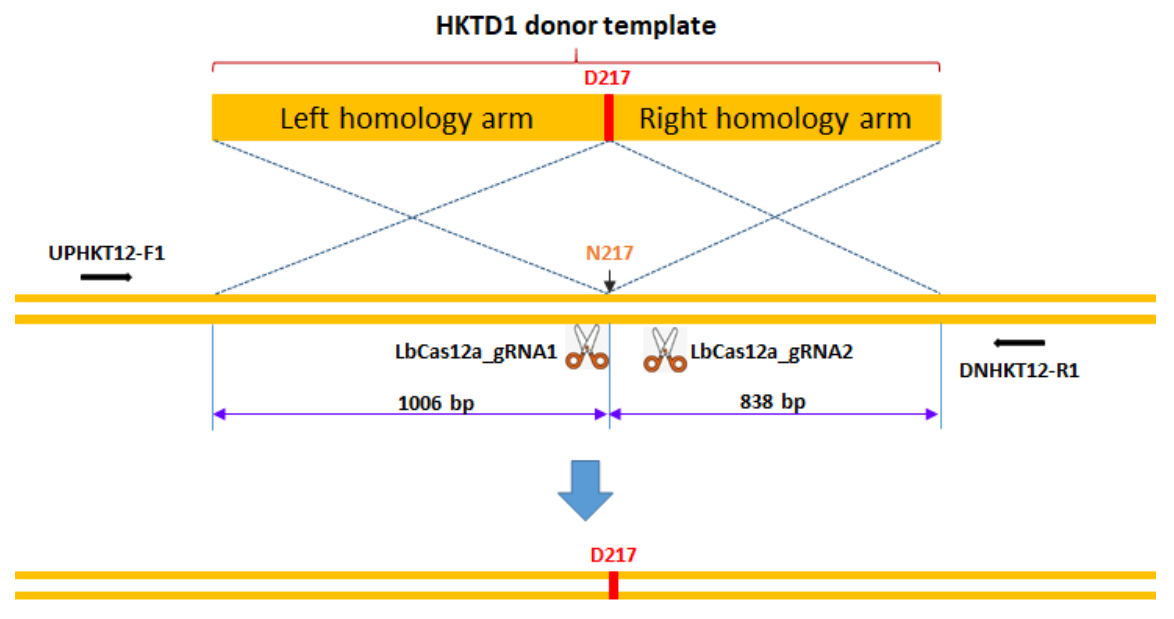

Precisely edited GT product

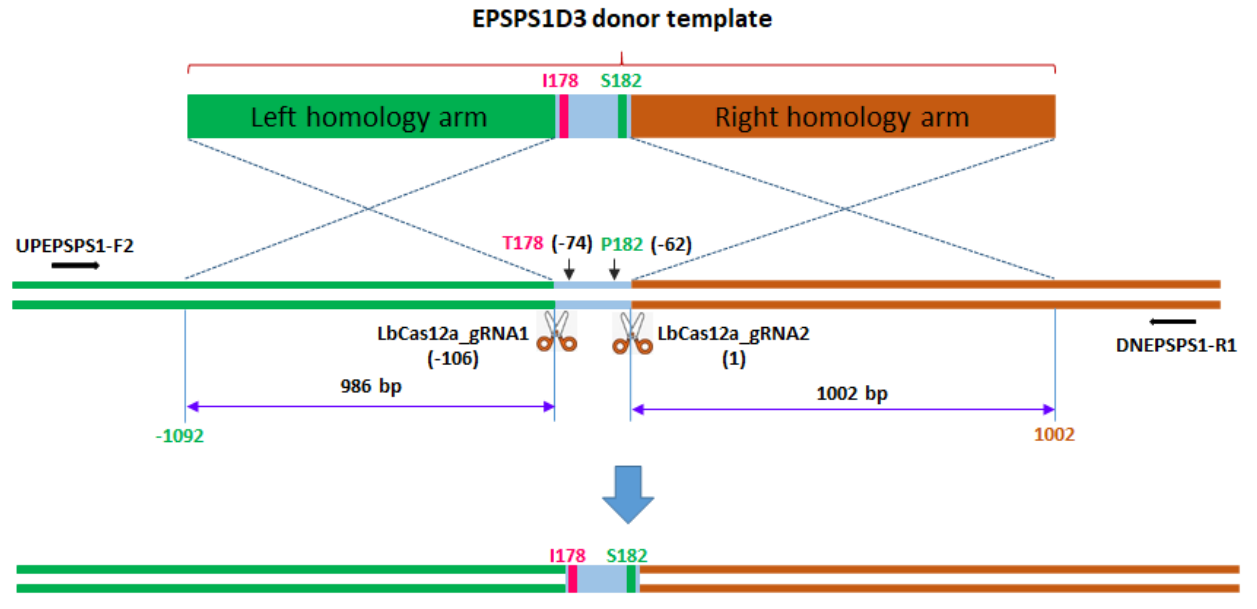

Precisely edited GT product

C

\begin{tabular}{|c|c|c|c|c|c|c|c|c|c|c|c|}
\hline \multirow[b]{2}{*}{$\begin{array}{c}\text { Targeted } \\
\text { gene }\end{array}$} & \multirow[b]{2}{*}{ Cargo } & \multirow[b]{2}{*}{$\begin{array}{c}\text { LbCas12a } \\
\text { variant }\end{array}$} & \multirow[b]{2}{*}{ crRNA } & \multicolumn{4}{|c|}{ Replicate 1} & \multicolumn{4}{|c|}{ Replicate 2} \\
\hline & & & & Total read & $\begin{array}{c}\text { GT } \\
\text { efficiency } \\
(\%)\end{array}$ & $\begin{array}{c}\text { Indel rate } \\
\text { of gRNA1 } \\
(\%)\end{array}$ & $\begin{array}{c}\text { Indel rate } \\
\text { of gRNA2 } \\
(\%)\end{array}$ & Total read & $\begin{array}{c}\text { GT } \\
\text { efficiency } \\
(\%)\end{array}$ & $\begin{array}{c}\text { Indel rate } \\
\text { of gRNA1 } \\
(\%)\end{array}$ & $\begin{array}{c}\text { Indel rate } \\
\text { of gRNA2 } \\
(\%)\end{array}$ \\
\hline \multirow{7}{*}{ SIHKT1;2 } & Replicon & wt & $\operatorname{crR} 1.20^{\mathrm{HKT} 1 ; 2}$ & 71070 & 0.000 & 0.74 & - & 46888 & 0.000 & 2.52 & - \\
\hline & Replicon & wt & $\operatorname{crR} 2.20^{\mathrm{HKT} 1 ; 2}$ & 51658 & 0.000 & - & 0.24 & 49471 & 0.000 & - & 1.51 \\
\hline & T-DNA & wt & $\operatorname{crR} 1-2.20^{\mathrm{HKT} 1 ; 2}$ & 58514 & 0.000 & 0.09 & 0.04 & 51889 & 0.000 & 0.52 & 0.17 \\
\hline & Replicon & wt & $\operatorname{crR} 1-2.20^{\mathrm{HKT} 1 ; 2}$ & 46531 & 0.000 & 0.69 & 0.35 & 50341 & 0.002 & 4.32 & 2.56 \\
\hline & Replicon & $\mathrm{tt}$ & $\operatorname{crR} 1-2.20^{\mathrm{HKT} 1 ; 2}$ & 43205 & 0.000 & 0.90 & 0.67 & 44029 & 0.005 & 3.87 & 2.39 \\
\hline & Replicon & wt & $\operatorname{crR} 1-2.23^{\mathrm{HKT1} ; 2}$ & 26965 & 0.004 & 3.30 & 1.06 & 33146 & 0.010 & 2.91 & 0.62 \\
\hline & Replicon & tt & $\operatorname{crR} 1-2.23^{\mathrm{HKT1} ; 2}$ & 20319 & 0.000 & 1.72 & 0.90 & 43393 & 0.000 & 1.49 & 0.71 \\
\hline \multirow{2}{*}{ SIEPSPS1 } & Replicon & wt & crR1-2.23 EPSPS1 & 59007 & 0.003 & 0.31 & - & 72638 & 0.006 & 1.89 & - \\
\hline & Replicon & $\mathrm{tt}$ & $\operatorname{crR} 1-2.23^{\mathrm{EPSPS} 1}$ & 40357 & 0.005 & 0.14 & - & 62013 & 0.010 & 1.41 & - \\
\hline
\end{tabular}


Fig. 4 GT performance of the LbCas12a variants at the SIHKT1;2 and SIEPSPS1 loci. a-b. Schematic diagrams describing the expected GT processes for exchanging the homologous DNA donor template with the genomic sequence at the SIHKT1;2 (a) and SIEPSPS1 (b) loci. The D217 coding sequence was added during the cloning of the HKTD1 donor for exchange with the N217 sequence of the genomic site. The lengths of homologous arms are shown. Two cutting sites (LbCas12a cutting sites 1 and 2) were planned to support the GT. The reverse and forward primers for amplifying the targeted sites by PCR are shown with black arrows. The I178 and S182 coding sequences were added during the cloning of the EPSPS1D3 donor for exchange with the T178 and P182 sequences of the genomic site. The lengths of homologous arms are shown. Two cutting sites (LbCas12a cutting sites 1 and 2 ) were used for the GT experiments. The reverse and forward primers for amplifying the targeted sites by PCR are shown with black arrows. In b, LbCas12a cutting site 2 is set as position 1, and the other positions are calculated accordingly. The diagrams were drawn not to their actual scales. c. The GT and indel mutation efficiencies assessed by targeted deep sequencing. At the SIHKT1;2

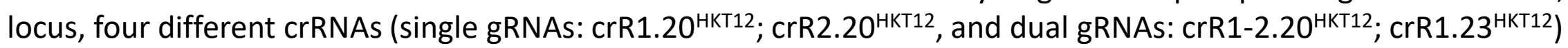
were used for comparison of the LbCas12a variants in GT performance. A T-DNA vector was also used for comparison with the replicon system. With the SIEPSPS1 gene, only one dual gRNA construct (crR1-2.23 $3^{\text {EPSPS1}}$ ) was used with the two LbCas12a variants. Wt: wild-type LbCas12a; tt: ttLbCas12a. 


\section{Figure 5}

\begin{tabular}{|c|c|c|c|c|c|c|c|c|}
\hline pHRES2.9 & & crR1-3.23 ${ }^{\text {EPSPS1 }}$ & 117670 & $0.002 \pm 0.002$ & $0.958 \pm 0.203$ & 149673 & $0.009 \pm 0.002$ & $1.339 \pm 0.233$ \\
\hline pHRES2.10 & wt & $\begin{array}{l}\text { crR1-3.23 } 3^{\text {EPSPS1 }} \\
+\mathrm{crR} 2-4.23^{\text {EPSPS1 }}\end{array}$ & 105753 & 0.000 & $0.934 \pm 0.042$ & 148166 & $0.004 \pm 0.003$ & $1.235 \pm 0.079$ \\
\hline pHRES2.12 & tt & $\begin{array}{c}\mathrm{crR} 1-3.23^{\mathrm{EPSPS} 1} \\
+\mathrm{crR} 2-4.23^{\mathrm{EPSPS} 1}\end{array}$ & 107335 & $0.002 \pm 0.002$ & $1.303 \pm 0.274$ & 70779 & $0.007 \pm 0.002$ & $1.867 \pm 0.283$ \\
\hline
\end{tabular}

b
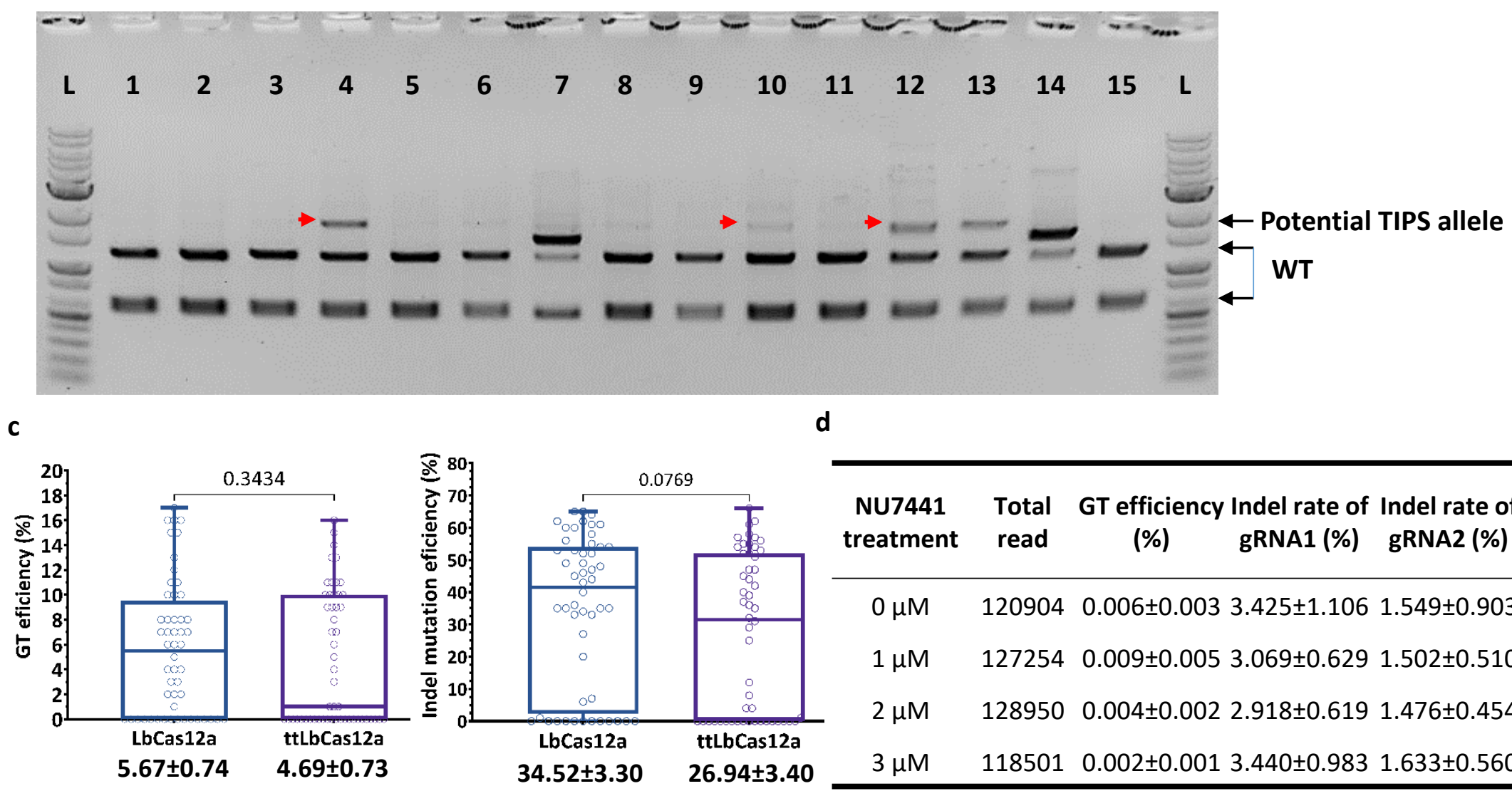

\begin{tabular}{cccccc}
\hline $\begin{array}{c}\text { NU7441 } \\
\text { treatment }\end{array}$ & $\begin{array}{c}\text { Total } \\
\text { read }\end{array}$ & $\begin{array}{c}\text { GT efficiency } \\
\text { (\%) }\end{array}$ & $\begin{array}{c}\text { Indel rate of } \\
\text { gRNA1 (\%) }\end{array}$ & $\begin{array}{c}\text { Indel rate of } \\
\text { gRNA2 (\%) }\end{array}$ \\
\hline $0 \mu \mathrm{M}$ & 120904 & $0.006 \pm 0.003$ & $3.425 \pm 1.106$ & $1.549 \pm 0.903$ \\
$1 \mu \mathrm{M}$ & 127254 & $0.009 \pm 0.005$ & $3.069 \pm 0.629$ & $1.502 \pm 0.510$ \\
$2 \mu \mathrm{M}$ & 128950 & $0.004 \pm 0.002$ & $2.918 \pm 0.619$ & $1.476 \pm 0.454$ \\
$3 \mu \mathrm{M}$ & 118501 & $0.002 \pm 0.001$ & $3.440 \pm 0.983$ & $1.633 \pm 0.560$ \\
\hline
\end{tabular}


Fig. 5 Further assessment of GT performance of the LbCas12a variants at the SIEPSPS1 locus. a. Assessment of GT efficiency by targeted deep sequencing with GT tools using two or four cutting sites with LbCas12a variants at the SIEPSPS1 locus. b-c. Indel mutation and GT efficiencies obtained with the LbCas12a variants at the plant stage. Fiftytwo plants of each LbCas12a variant were obtained from the transformation of the GT tool with the crR1-2.23 EPSPS1 expression cassette and used for PCR amplification of the targeted site with the UPEPSPS1-F2 and DNEPSPS1-R1 primers. In $\mathbf{b}$, the PCR products were purified and screened for the potential GT allele by Bpil digestion since the Bpil site near the targeted site was modified in the DNA donor sequence. The red arrows indicate potential GT bands. 1-15: Representative transformants obtained from the transformation using the GT construct containing LbCas12a and crR13.23 ${ }^{\text {EPSPS1. In }}$ c: All the purified PCR products were sequenced by the Sanger method, and the ab1 files were subsequently analyzed by ICE Synthego software to reveal the indel mutation and GT efficiencies. The indel mutation and GT efficiencies of all the samples were statistically analyzed using Student's t-test and plotted by GraphPad Prism version 9. The editing efficiencies (mean \pm SEM) are shown at the bottom of each box. $\mathbf{d}$. Targeted deep sequencingmediated evaluation of NU7441 impacts on ttLbCas12a-based GT efficiency. 